\title{
Nanofluids flow over a permeable unsteady stretching surface with non-uniform heat source/sink in the presence of inclined magnetic field
}

\author{
N. S. Elgazery
}

Correspondence: nasersaleh@edu. asu.edu.eg

Department of Mathematics, Faculty of Education, Ain Shams University, El Makrizy Street, Roxy, Heliopolis, Cairo 11341, Egypt

\begin{abstract}
This work analyzes the unsteady two-dimensional nanofluid flow over a vertical stretching permeable surface in the presence of an inclined magnetic field and nonuniform heat source/sink. Four different types of nanoparticles, namely silver Ag, copper $\mathrm{Cu}$, alumina $\mathrm{Al}_{2} \mathrm{O}_{3}$, and titania $\mathrm{TiO}_{2}$, are considered by using water as a base fluid with the Prandtl number $\operatorname{Pr}=6.785$. The governing partial differential equations are transformed to coupled non-linear ordinary differential equations by appropriate similarity transformation. Furthermore, the similarity equations are solved numerically by using the fourth-order Runge-Kutta integration scheme with Newton Raphson shooting method. A comparison of obtained numerical results is made with previously published results in some special cases, and excellent agreement is noted. Numerical results for velocity and temperature profiles as well as skin friction coefficient and local Nusselt number are discussed for various values of physical parameters. It tends to be discovered that, the magnetic field inclination angle $\gamma$ has the capability to strengthens the magnetic field and reduce the velocity profile of the flow. Also, it can be found that, by using various types of nanofluids, velocity and temperature distributions change, which means that the nanofluids are important in the cooling and heating processes. The thermal boundary layer thickness is related to the increased thermal conductivity of different types of nanofluids, i.e., the minimum (maximum) value of the temperature is obtained by adding titanium oxide (silver) to the fluid as the nanoparticles.
\end{abstract}

Keywords: Nanofluids, Inclined magnetic field, Unsteady stretching permeable surface, Non-uniform heat source/sink

JEL Classification: C39, C63, C88

\section{Introduction}

The study of hydrodynamic flow and heat transfer over a stretching sheet has gained considerable attention due to its vast applications in industry and its importance to several technological processes. Sakiadis [1] was the first initiate such a problem by considering the boundary layer fluid flow over a continuous solid surface moving with a constant velocity. The thermal behavior of the problem was studied by Erickson

(c) The Author(s). 2019 Open Access This article is distributed under the terms of the Creative Commons Attribution 4.0 International License (http://creativecommons.org/licenses/by/4.0/), which permits unrestricted use, distribution, and reproduction in any medium, provided you give appropriate credit to the original author(s) and the source, provide a link to the Creative Commons license, and indicate if changes were made. 
et al. [2] and experimentally verified by Tsou et al. [3]. Crane [4] extended the work of Sakiadis [1] to the flow caused by an elastic sheet moving in its own plane with a velocity varying linearly with the distance from a fixed point. Then, several investigators [5-8] studied the steady or unsteady boundary layer flow of Newtonian and non-Newtonian fluids over linear and nonlinear stretching surfaces.

The word "nano" dates back to 1915 in the book The World of Neglected Dimensions by Oswald [9]. A unique feature of the matter at nano-scale makes nanotechnology a new research territory in the twenty-first century. In the last few decades, scientists and researchers surrounding the globe tried to continuously work on various aspects of nanotechnology. Nanofluid is described as a fluid in which solid nanoparticles with the length scales of $1-100 \mathrm{~nm}$ are suspended in conventional heat transfer basic fluid. These nanoparticles enhance thermal conductivity and the convective heat transfer coefficient of the base fluid significantly [10-12]. Conventional heat transfer fluids such as oil, water, and ethylene glycol mixture are poor heat transfer fluids, because the thermal conductivity of these fluids affects the heat transfer coefficient between the heat transfer medium and the heat transfer surface. In 1995, Choi [13] became the first person to use the term "nanofluid" to describe a fluid containing nanoparticles. Choi et al. [14] showed that the addition of a small amount (less than $1 \%$ by volume) of nanoparticles to conventional heat transfer liquids increased the thermal conductivity of the fluid up to approximately two times. Many investigators studied the various characteristics of fluid flow and heat transfer behavior of nanofluids over the past 20 years [15-17] and found that enhanced heat transfer coefficients were obtained with nanofluids. Experimental studies $[18,19]$ showed that even with a small volumetric fraction of nanoparticles (usually less than 5\%), the thermal conductivity of the base fluid is enhanced by $10-50 \%$ with a remarkable improvement in the convective heat transfer coefficient.

The study of magnetic field effects has important applications in physics, chemistry, and engineering. Many industrial types of equipment, such as the magneto hydrodynamic (MHD) generator, pumps, bearings, and boundary layer control, are affected by the interaction between the electrically conducting fluid and a magnetic field. Recently, the works of many investigators studied the influences of electrically conducting in the presence of magnetic fields for nanofluids. These studies have important applications in physics, chemistry, biomedical engineering, biosciences, and nuclear power plants. One of the basic and important problems in this area is the unsteady magnetic nanofluid behavior of boundary layers along fixed or moving stretching permeable surfaces. Daniel et al. [20] studied double stratification effects on unsteady electrical MHD mixed convection flow of nanofluid with viscous dissipation and Joule heating. The MHD heat and mass transfer flow of a nanofluid over an inclined vertical porous plate with radiation and heat generation/absorption was studied by Reddy et al. [21]. Prabhavathi et al. [22] and Sreedevi et al. [23] studied the MHD boundary layer heat and mass transfer flow over a vertical cone embedded in a porous media filled with nanofluid. Hayat et al. [24] presented numerical simulation for melting heat transfer and radiation effects in stagnation point flow of a carbon-water nanofluid. All these studies were concerned with Newtonian fluid flows, whereas mere studies for the MHD non-Newtonian nanofluid flow induced by a stretching sheet were analyzed by Madhu et al. [25], Hayat et al. [26], and Hsiao [27]. Eldabe and Abou-zeid [28] presented the homotopy perturbation method for MHD pulsatile non-Newtonian nanofluid flow with heat transfer 
through a non-Darcy porous medium. Sreedevi et al. [29] studied heat and mass transfer analysis of nanofluid over a linear and non-linear stretching surface with thermal radiation and chemical reaction. Also, magneto-hydrodynamics heat and mass transfer analysis of single and multi-wall carbon nanotubes over a vertical cone with convective was studied recently by the same authors [30]. Jyothi et al. [31] studied an influence of magnetic field and thermal radiation on convective flow of SWCNTs-water and MWCNTs-water nanofluid between rotating stretchable disks with convective boundary conditions. Analysis of activation energy in Couette-Poiseuille flow of nanofluid in the presence of chemical reaction and convective boundary conditions was studied by Zeeshan et al. [32].

In view of the above studies, it is evident, to the best of authors' knowledge, that no attempts have far been instigated with regard to studying inclined magnetohydrodynamic nanofluid transport with non-uniform heat source/sink effects. Then the aim of the present work is studying the effects of nanoparticles on the heat transfer characteristics over a permeable unsteady stretching sheet. Furthermore, in the present model, the supplementary effects of non-uniform heat source/sink and inclined magnetic field are also considered. In the present study, the nanofluid model proposed by Tiwari and Das [33] was used, as this model successfully applied in several papers [34-36]. Through an appropriate similarity transformation, the governing partial differential equations are reduced into ordinary differential equations, which are solved numerically by using the fourth-order Runge-Kutta integration scheme with the Newton Raphson shooting method with the help of the symbolic computational software MATHEMATICA. A comparison of obtained numerical results is made with previously published results [37-39] in some special cases, and excellent agreement is noted. The obtained results are shown graphically, and the physical aspects of the problem are discussed. Also, the skin friction coefficient and the local Nusselt number at the stretching surface are displayed in a tabular form and discussed.

\section{Mathematical formulation}

Consider unsteady, two-dimensional, laminar, incompressible, and electrically conducting boundary layer flow due to a permeable stretching vertical sheet, where the sheet is along the $x$ direction. Figure 1 shows the coordinate system and flow model. In a water-based nanofluid, different types of nanoparticles, $\mathrm{Ag}, \mathrm{Cu}, \mathrm{Al}_{2} \mathrm{O}_{3}$, and $\mathrm{TiO}_{2}$, are contained. A uniform inclined magnetic field $B_{0}$ is applied to the flow [40]. An inclined magnetic field with an acute angle $(\gamma)$ is applied along the $x$ direction. The inclination angles $0^{\circ}, 90^{\circ}$, and $0^{\circ}<\gamma<90^{\circ}$ represent the horizontal, vertical, and inclined magnetic field scenarios respectively. At $\gamma=0^{\circ}$, the magnetic field vanishes (because $\sin 0=0$ ), and at $\gamma=\pi / 2$, this magnetic field acts like a transverse magnetic field along the $y$ axis (because $\sin \pi / 2=1$ ). It is also assumed that the induced magnetic field and viscous dissipation effects are negligible. The convective heat transfer is taken into account. The flow is caused by the linear stretching of the sheet which moves in its own plane with the surface velocity $U_{w}(x, t)=\frac{a x}{1-c t}$, where $a$ (stretching rate) and $c$ are positive constants having dimension (time) ${ }^{-1}$ (with $c t<1, c \geq 0$ ) [37]. The temperature $T_{w}(x, t)$ at the sheet surface is considered as a function of the distance $x$ and time $t$ which is also higher than the ambient fluid temperature $T_{\infty}$. It is also assumed that the base fluid 


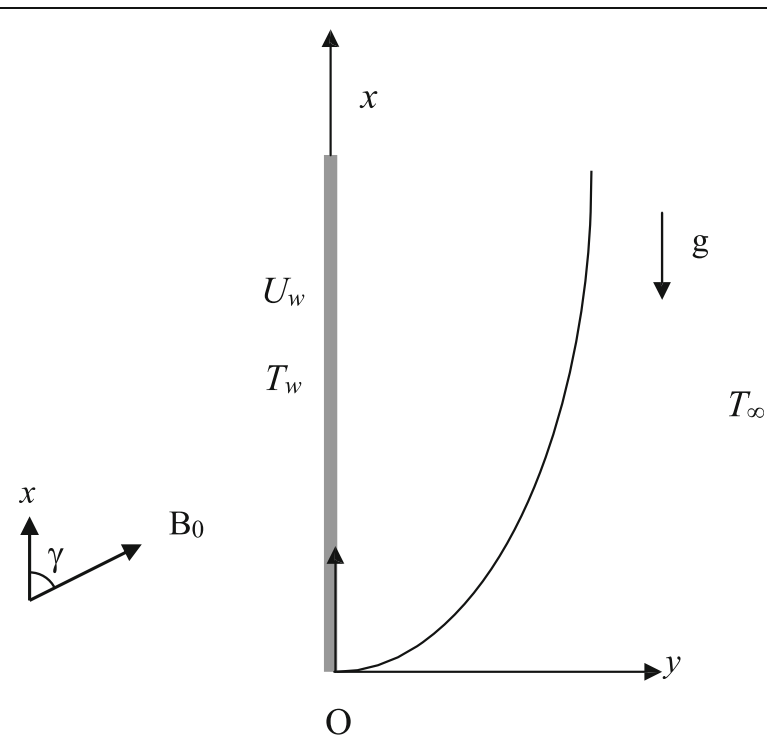

Fig. 1 The physical model and coordinate system

(i.e., water) and the nanoparticles are in thermal equilibrium and no slip occurs between them. The thermophysical properties of the nanofluids are given in Table 1 [35]. The boundary layer equations that govern the present flow and heat transfer for a nanofluid in the presence of inclined magnetic field and internal heat generation/absorption subject to the Boussinesq approximations can be expressed as

$$
\begin{aligned}
& \frac{\partial u}{\partial x}+\frac{\partial v}{\partial y}=0 \\
& \frac{\partial u}{\partial t}+u \frac{\partial u}{\partial x}+v \frac{\partial u}{\partial y}=\frac{\mu_{n f}}{\rho_{n f}} \frac{\partial^{2} u}{\partial y^{2}}+g \frac{(\rho \beta)_{n f}}{\rho_{n f}}\left(T-T_{\infty}\right)-\frac{\sigma_{n f}}{\rho_{n f}} B_{0}^{2} \sin ^{2} \gamma u \\
& \frac{\partial T}{\partial t}+u \frac{\partial T}{\partial x}+v \frac{\partial T}{\partial y}=\alpha_{n f} \frac{\partial^{2} T}{\partial y^{2}}+\frac{q^{\prime \prime \prime}}{\left(\rho C_{P}\right)_{n f}}
\end{aligned}
$$

Here, $u$ and $v$ are the velocity components along the $x$ and $y$ axes respectively. The boundary conditions for the velocity components at the surface and temperature can be written as [37]

Table 1 Thermophysical properties of water and nanoparticles

\begin{tabular}{lllll}
\hline & $\rho\left(\mathrm{kg} / \mathrm{m}^{3}\right)$ & $C p(\mathrm{~J} / \mathrm{kg} \mathrm{K})$ & $k(\mathrm{~W} / \mathrm{m} \mathrm{K})$ & $\beta \times 10^{-5}\left(\mathrm{~K}^{-1}\right)$ \\
\hline Pure water & 997.1 & 4179 & 0.613 & 21 \\
Copper $(\mathrm{Cu})$ & 8933 & 385 & 401 & 1.67 \\
Silver $(\mathrm{Ag})$ & 10,500 & 235 & 429 & 1.89 \\
Alumina $\left(\mathrm{Al}_{2} \mathrm{O}_{3}\right)$ & 3970 & 765 & 40 & 0.85 \\
Titanium oxide $\left(\mathrm{TiO}_{2}\right)$ & 4250 & 686.2 & 8.9538 & 0.9 \\
\hline
\end{tabular}


Table 2 Values of $-\theta^{\prime}(0)$ for various values of $S, \lambda$, and $\operatorname{Pr}$ (the error $=$ exact solutions - present results)

\begin{tabular}{|c|c|c|c|c|c|c|c|c|}
\hline$S$ & $\lambda$ & $\operatorname{Pr}$ & Grubka and Bobba & Ali & $\begin{array}{l}\text { Exact solutions } \\
\text { Ishak at el. }\end{array}$ & Ishak at el. & Present results & The error \\
\hline \multirow[t]{10}{*}{0} & 0 & 0.01 & 0.0197 & & 0.01970635421 & 0.0197 & 0.01967 & $3.63542 \times 10^{-5}$ \\
\hline & & 0.72 & 0.8086 & 0.8058 & 0.8086313498 & 0.8086 & 0.80863 & $1.3498 \times 10^{-5}$ \\
\hline & & 1.0 & 1.0000 & 0.9961 & 1.0000000000 & 1.0000 & 1.00001 & $1 \times 10^{-5}$ \\
\hline & & 3.0 & 1.9237 & 1.9144 & 1.923682594 & 1.9237 & 1.92368 & $2.594 \times 10^{-6}$ \\
\hline & & 7.0 & & & 3.072250207 & 3.0723 & 3.07224 & $1.0207 \times 10^{-5}$ \\
\hline & & 10 & 3.7207 & 3.7006 & 3.720673901 & 3.7207 & 3.72066 & $1.3901 \times 10^{-5}$ \\
\hline & & 100 & 12.2940 & & 12.29408326 & 12.2941 & 12.29408 & $3.26 \times 10^{-6}$ \\
\hline & 1 & 1 & & & & 1.0873 & 1.08728 & \\
\hline & 2 & & & & & 1.1423 & 1.14225 & \\
\hline & 3 & & & & & 1.1853 & 1.18526 & \\
\hline \multirow[t]{4}{*}{1} & 0 & & & & & 1.6820 & 1.68199 & \\
\hline & 1 & & & & & 1.7039 & 1.70391 & \\
\hline & -0.5 & 10 & & & & 5.5585 & 5.55852 & \\
\hline & 0.5 & & & & & 5.5690 & 5.56901 & \\
\hline \multicolumn{9}{|c|}{$u=U_{w}(x, t)=\frac{a x}{1-c t}$} \\
\hline & $u=0$ & & $T=T_{\infty}$ & & & & & as $y \rightarrow \infty$ \\
\hline
\end{tabular}

Here, $f_{w}>0$ and $f_{w}<0$ correspond to suction and injection parameters, $b$ is a constant and has a dimension temperature/length. $b>0$ and $b<0$ correspond to assisting and opposing flows, and $b=0$ is for forced convection limit (absence of buoyancy force).

\section{Thermophysical properties of nanofluid}

The density of the nanofluid $\rho_{n f}$, electrical conductivity of the nanofluid $\sigma_{n f}$, thermal diffusivity of the nanofluid $\alpha_{n f}$, heat capacity $C_{B}$ and the thermal expansion coefficient $\beta$ are presented as follows, respectively

$$
\left.\begin{array}{c}
\rho_{n f}=(1-\phi) \rho_{f}+\phi \rho_{P}, \quad \sigma_{n f}=(1-\phi) \sigma_{f}+\phi \sigma_{P}, \\
\left(\rho C_{P}\right)_{n f}=(1-\phi)\left(\rho C_{P}\right)_{f}+\phi\left(\rho C_{P}\right)_{P}, \quad(\rho \beta)_{n f}=(1-\phi)(\rho \beta)_{f}+\phi(\rho \beta)_{P}
\end{array}\right\}
$$

where $\phi$ is the nanoparticle volume fraction, $\rho_{f}$ is the reference density of the fluid fraction, $\rho_{P}$ is the reference density of the solid fraction, $\sigma_{f}$ is the reference electrical conductivity of the fluid fraction, and $\sigma_{P}$ is the reference electrical conductivity of the solid fraction. Also, $\left(C_{P}\right)_{f}$ is the reference heat capacity of the fluid fraction, $\left(C_{P}\right)_{P}$ is the reference heat capacity of the solid fraction, $(\beta)_{f}$ is the reference thermal expansion coefficient of the fluid fraction, and $(\beta)_{P}$ is the reference thermal expansion coefficient of the solid fraction.

The effective dynamic viscosity of nanofluid, $\mu_{n f}$, is given by the Brinkman model [41] as 
Table 3 Values of $-f^{\prime \prime}(0)$ and $-\theta^{\prime}(0)$ for various $A, B$, and $\varphi$ with $\gamma=\frac{\pi}{3}, \operatorname{Pr}=6.785, \lambda=0.5$, and $S=M=f_{w}=0.1$

\begin{tabular}{|c|c|c|c|c|c|c|c|c|c|c|}
\hline \multirow[t]{2}{*}{$A$} & \multirow[t]{2}{*}{ B } & \multirow[t]{2}{*}{$\varphi$} & \multicolumn{4}{|l|}{$-f^{\prime \prime}(0)$} & \multicolumn{4}{|l|}{$-\theta^{\prime}(0)$} \\
\hline & & & $\mathrm{Cu}$ & $\mathrm{Ag}$ & $\mathrm{Al}_{2} \mathrm{O}_{3}$ & $\mathrm{TiO}_{2}$ & $\mathrm{Cu}$ & $\mathrm{Ag}$ & $\mathrm{Al}_{2} \mathrm{O}_{3}$ & $\mathrm{TiO}_{2}$ \\
\hline \multirow[t]{6}{*}{-0.5} & -0.5 & 0.05 & 1.13901 & 1.17511 & 1.02053 & 1.02853 & 3.47169 & 3.45867 & 3.45288 & 3.50071 \\
\hline & & 0.15 & 1.26227 & 1.33798 & 1.00119 & 1.02005 & 2.84113 & 2.81764 & 2.78241 & 2.90208 \\
\hline & 0.0 & 0.05 & 1.13772 & 1.17374 & 1.01947 & 1.0275 & 3.37271 & 3.3534 & 3.37184 & 3.41978 \\
\hline & & 0.15 & 1.26009 & 1.33549 & 0.999799 & 1.0188 & 2.69323 & 2.6504 & 2.68589 & 2.80694 \\
\hline & 0.5 & 0.05 & 1.13637 & 1.17229 & 1.01838 & 1.02645 & 3.27066 & 3.24457 & 3.2888 & 3.33687 \\
\hline & & 0.15 & 1.25767 & 1.33261 & 0.998347 & 1.0175 & 2.53558 & 2.4697 & 2.5857 & 2.70841 \\
\hline \multirow[t]{6}{*}{0.0} & -0.5 & 0.05 & 1.12971 & 1.16571 & 1.01175 & 1.0199 & 3.27349 & 3.25111 & 3.2836 & 3.33095 \\
\hline & & 0.15 & 1.25236 & 1.32806 & 0.992261 & 1.0116 & 2.59005 & 2.54533 & 2.54533 & 2.71881 \\
\hline & 0.0 & 0.05 & 1.12769 & 1.16356 & 1.01013 & 1.01833 & 3.16522 & 3.13552 & 3.19592 & 3.24342 \\
\hline & & 0.15 & 1.24869 & 1.32384 & 0.989996 & 1.00957 & 2.42212 & 2.35369 & 2.49284 & 2.614 \\
\hline & 0.5 & 0.05 & 1.12549 & 1.16119 & 1.0084 & 1.01665 & 3.05233 & 3.01454 & 3.10533 & 3.15306 \\
\hline & & 0.15 & 1.24412 & 1.31831 & 0.987451 & 1.0073 & 2.23725 & 2.13799 & 2.38041 & 2.50385 \\
\hline \multirow[t]{6}{*}{0.5} & -0.5 & 0.05 & 1.12028 & 1.15619 & 1.00285 & 1.01116 & 3.07389 & 3.04207 & 3.1132 & 3.1601 \\
\hline & & 0.15 & 1.24223 & 1.31792 & 0.983153 & 1.003 & 2.33646 & 2.27023 & 2.41486 & 2.53408 \\
\hline & 0.0 & 0.05 & 1.11753 & 1.15325 & 1.00065 & 1.00903 & 2.95612 & 2.91593 & 3.01872 & 3.06584 \\
\hline & & 0.15 & 1.23698 & 1.31188 & 0.979972 & 1.00014 & 2.14762 & 2.05304 & 2.29781 & 2.41928 \\
\hline & 0.5 & 0.05 & 1.11447 & 1.14996 & 0.998258 & 1.00671 & 2.83218 & 2.78256 & 2.92044 & 2.96785 \\
\hline & & 0.15 & 1.23015 & 1.30354 & 0.97628 & 0.996866 & 1.93409 & 1.80031 & 2.17266 & 2.29711 \\
\hline
\end{tabular}

$$
\mu_{n f}=\frac{\mu_{f}}{(1-\phi)^{2.5}}
$$

Also, the effective thermal conductivity of nanofluid, $k_{n f}$ is given by the Maxwell model [42] as

Table 4 Values of $-f^{\prime \prime}(0)$ and $-\theta^{\prime}(0)$ for various $M, f_{w}$ and $\varphi$ with $\gamma=\pi / 3, \operatorname{Pr}=6.785, A=B=\lambda=0.5$,

\begin{tabular}{|c|c|c|c|c|c|c|c|c|c|c|}
\hline \multirow[t]{2}{*}{$\bar{M}$} & \multirow[t]{2}{*}{$f_{w}$} & \multirow[t]{2}{*}{$\varphi$} & \multicolumn{4}{|l|}{$-f^{\prime \prime}(0)$} & \multicolumn{4}{|l|}{$-\theta^{\prime}(0)$} \\
\hline & & & $\overline{\mathrm{Cu}}$ & $\mathrm{Ag}$ & $\mathrm{Al}_{2} \mathrm{O}_{3}$ & $\mathrm{TiO}_{2}$ & $\overline{\mathrm{Cu}}$ & $\mathrm{Ag}$ & $\mathrm{Al}_{2} \mathrm{O}_{3}$ & $\mathrm{TiO}_{2}$ \\
\hline \multirow[t]{2}{*}{0.0} & -0.4 & 0.2 & 0.889971 & 0.936099 & 0.717616 & 0.735 & 0.905225 & 0.762394 & 1.17911 & 1.25589 \\
\hline & 0.4 & & 1.49746 & 1.61218 & 1.10429 & 1.13512 & 2.06511 & 1.86895 & 2.37644 & 2.6085 \\
\hline \multirow[t]{2}{*}{0.1} & -0.4 & & 0.890826 & 0.936731 & 0.719322 & 0.736626 & 0.905244 & 0.76228 & 1.17871 & 1.25549 \\
\hline & 0.4 & & 1.51025 & 1.62575 & 1.11638 & 1.14704 & 2.07879 & 1.88918 & 2.38022 & 2.61235 \\
\hline \multirow[t]{2}{*}{0.2} & -0.4 & & 0.893492 & 0.938693 & 0.724402 & 0.741468 & 0.905512 & 0.762256 & 1.17764 & 1.25444 \\
\hline & 0.4 & & 1.59632 & 1.68616 & 1.155 & 1.18494 & 2.28853 & 2.02818 & 2.39789 & 2.62955 \\
\hline \multirow[t]{2}{*}{0.0} & -0.4 & 0.0 & 0.711773 & 0.711773 & 0.711773 & 0.711773 & 1.80348 & 1.80348 & 1.80348 & 1.80348 \\
\hline & 0.4 & & 1.164690 & 1.164690 & 1.164690 & 1.164690 & 4.69970 & 4.69970 & 4.69970 & 4.69970 \\
\hline \multirow[t]{2}{*}{0.1} & -0.4 & & 0.714450 & 0.714450 & 0.714450 & 0.714450 & 1.80271 & 1.80271 & 1.80271 & 1.80271 \\
\hline & 0.4 & & 1.185340 & 1.185340 & 1.185340 & 1.185340 & 4.70416 & 4.70416 & 4.70416 & 4.70416 \\
\hline \multirow[t]{2}{*}{0.2} & -0.4 & & 0.722442 & 0.722442 & 0.722442 & 0.722442 & 1.80053 & 1.80053 & 1.80053 & 1.80053 \\
\hline & 0.4 & & 1.335620 & 1.335620 & 1.335620 & 1.335620 & 4.88937 & 4.88937 & 4.88937 & 4.88937 \\
\hline
\end{tabular}


Table 5 Values of $-f^{\prime \prime}(0)$ and $-\theta^{\prime}(0)$ for various $S, \lambda$ and $\varphi$ with $\gamma=\pi / 3, \operatorname{Pr}=6.785, A=B=0.5, M=$ $f_{w}=0.1$

\begin{tabular}{|c|c|c|c|c|c|c|c|c|c|c|}
\hline \multirow[t]{2}{*}{$S$} & \multirow[t]{2}{*}{$\lambda$} & \multirow[t]{2}{*}{$\varphi$} & \multicolumn{4}{|l|}{$-f^{\prime \prime}(0)$} & \multicolumn{4}{|l|}{$-\theta^{\prime}(0)$} \\
\hline & & & $\mathrm{Cu}$ & $\mathrm{Ag}$ & $\mathrm{Al}_{2} \mathrm{O}_{3}$ & $\mathrm{TiO}_{2}$ & $\mathrm{Cu}$ & $\mathrm{Ag}$ & $\mathrm{Al}_{2} \mathrm{O}_{3}$ & $\mathrm{TiO}_{2}$ \\
\hline \multirow[t]{3}{*}{0.0} & -0.5 & 0.2 & 1.41293 & 1.49943 & 1.10995 & 1.12069 & 1.29248 & 1.07747 & 1.65548 & 1.81398 \\
\hline & 0.0 & & 1.30072 & 1.3816 & 1.01055 & 1.02874 & 1.32189 & 1.11811 & 1.67487 & 1.83134 \\
\hline & 0.5 & & 1.19427 & 1.33082 & 0.914089 & 0.939062 & 1.34751 & 1.44053 & 1.69276 & 1.84759 \\
\hline \multirow[t]{3}{*}{1.0} & -0.5 & 0.2 & 1.74632 & 1.84716 & 1.37428 & 1.39378 & 2.67991 & 2.54474 & 2.86331 & 3.07003 \\
\hline & 0.0 & & 1.68021 & 1.78341 & 1.30777 & 1.33124 & 2.68307 & 2.54745 & 2.86727 & 3.07384 \\
\hline & 0.5 & & 1.6144 & 1.71993 & 1.24161 & 1.26898 & 2.68621 & 2.55013 & 2.87118 & 3.0776 \\
\hline \multirow[t]{3}{*}{2.0} & -0.5 & 0.2 & 2.0543 & 2.17411 & 1.61399 & 1.63891 & 3.52483 & 3.38637 & 3.67459 & 3.92463 \\
\hline & 0.0 & & 2.00255 & 2.12469 & 1.56063 & 1.58853 & 3.52624 & 3.38755 & 3.67663 & 3.92645 \\
\hline & 0.5 & & 1.95088 & 2.07534 & 1.50739 & 1.53826 & 3.52763 & 3.38871 & 3.67833 & 3.92826 \\
\hline \multirow[t]{3}{*}{0.0} & -0.5 & 0.0 & 1.174570 & 1.174570 & 1.174570 & 1.174570 & 3.12932 & 3.12932 & 3.12932 & 3.12932 \\
\hline & 0.0 & & 1.064010 & 1.064010 & 1.064010 & 1.064010 & 3.14750 & 3.14750 & 3.14750 & 3.14750 \\
\hline & 0.5 & & 0.955294 & 0.955294 & 0.955294 & 0.955294 & 3.16495 & 3.16495 & 3.16495 & 3.16495 \\
\hline \multirow[t]{3}{*}{1.0} & -0.5 & & 1.453830 & 1.453830 & 1.453830 & 1.453830 & 4.75737 & 4.75737 & 4.75737 & 4.75737 \\
\hline & 0.0 & & 1.371600 & 1.371600 & 1.371600 & 1.371600 & 4.76297 & 4.76297 & 4.76297 & 4.76297 \\
\hline & 0.5 & & 1.289750 & 1.289750 & 1.289750 & 1.289750 & 4.76851 & 4.76851 & 4.76851 & 4.76851 \\
\hline \multirow[t]{3}{*}{2.0} & -0.5 & & 1.703550 & 1.703550 & 1.703550 & 1.703550 & 5.94220 & 5.94220 & 5.94220 & 5.94220 \\
\hline & 0.0 & & 1.635490 & 1.635490 & 1.635490 & 1.635490 & 5.94506 & 5.94506 & 5.94506 & 5.94506 \\
\hline & 0.5 & & 1.567590 & 1.567590 & 1.567590 & 1.567590 & 5.94791 & 5.94791 & 5.94791 & 5.94791 \\
\hline
\end{tabular}

$$
\frac{k_{n f}}{k_{f}}=\frac{\left(k_{P}+2 k_{f}\right)-2 \phi\left(k_{f}-k_{P}\right)}{\left(k_{P}+2 k_{f}\right)+\phi\left(k_{f}-k_{P}\right)}
$$

Here, $\mu_{f}$ is the viscosity of fluid fraction, $v_{f}=\mu_{f} / \rho_{f}$ is the kinematic viscosity of fluid fraction, $k_{f}$ is the thermal conductivity of fluid, and $k_{P}$ is the thermal conductivity of solid.

\section{Dimensionless forms of equations}

By introducing the following non-dimensional variables

$$
\eta=\sqrt{\frac{a}{v_{f}(1-c t)}} y, \quad \psi(x, y, t)=\sqrt{\frac{a v_{f}}{(1-c t)}} x f(\eta), \quad \theta(\eta)=\frac{T-T_{\infty}}{T_{w}-T_{\infty}}
$$

Here, $\psi(x, y, t)$ is the stream function that is defined as

$$
u=\frac{\partial \psi}{\partial y}=\frac{a}{(1-c t)} x f^{\prime}(\eta) \text { and } v=-\frac{\partial \psi}{\partial x}=-\sqrt{\frac{a v_{f}}{(1-c t)}} f(\eta)
$$

The velocity components $u$ and $v$ in Eq. (9) automatically satisfy the continuity Eq. (1). It must be noted that expression (9) is valid only for $(c t<1)$. The non-uniform heat source/sink $q$ " 'is modeled as [43]

$$
q^{\prime \prime \prime}=\frac{U_{w}(x) k_{n f}}{x v_{n f}}\left[A\left(T_{w}-T_{\infty}\right) f^{\prime}(\eta)+B\left(T-T_{\infty}\right)\right]
$$

where $A$ and $B$ are the coefficients of space- and temperature-dependent heat source/ sink respectively. Note that $A>0$ and $B>0$ correspond to internal heat generation and $A<0$ and $B<0$ correspond to internal heat absorption. 


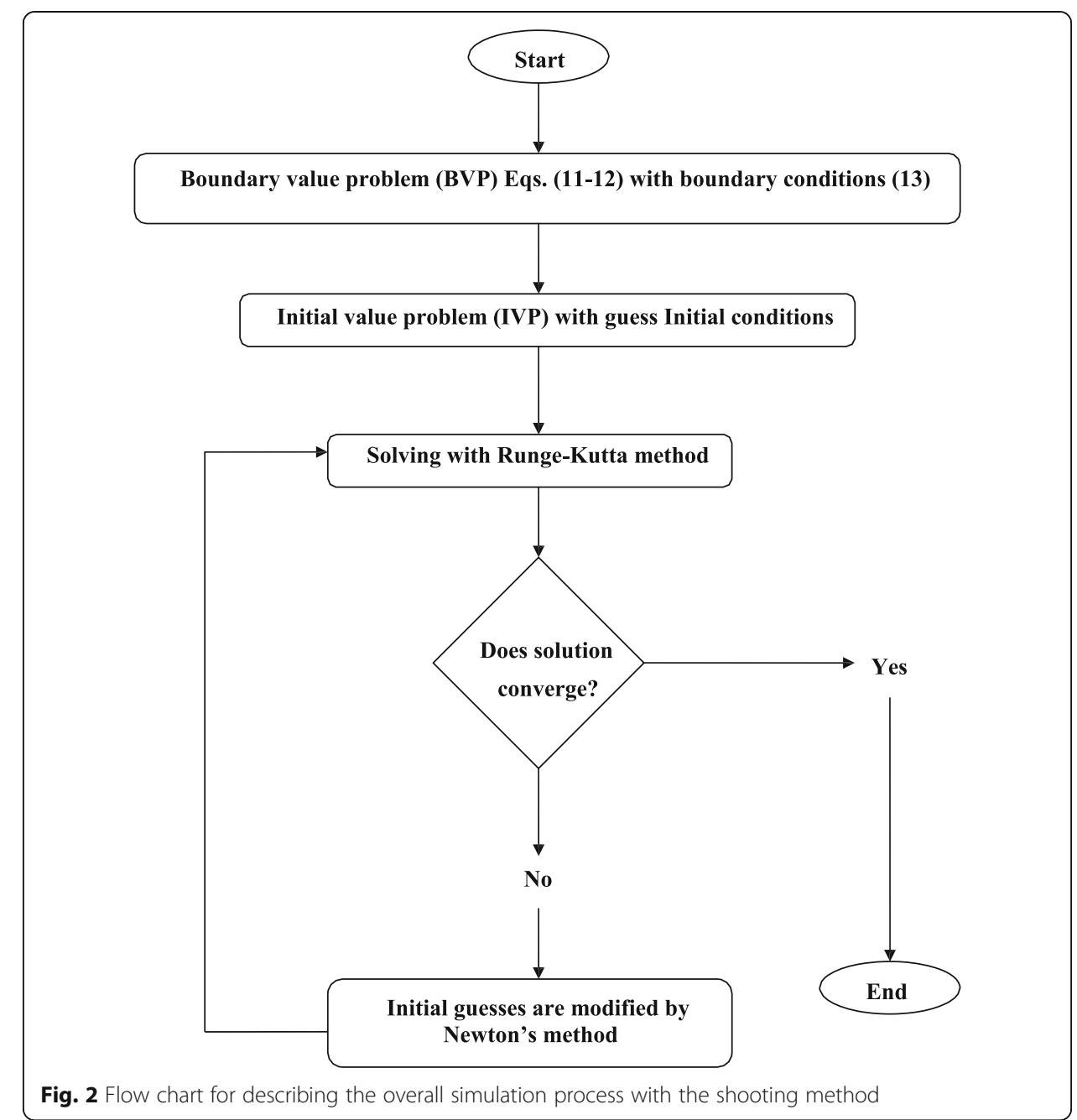

Fig. 2 Flow chart for describing the overall simulation process with the shooting method

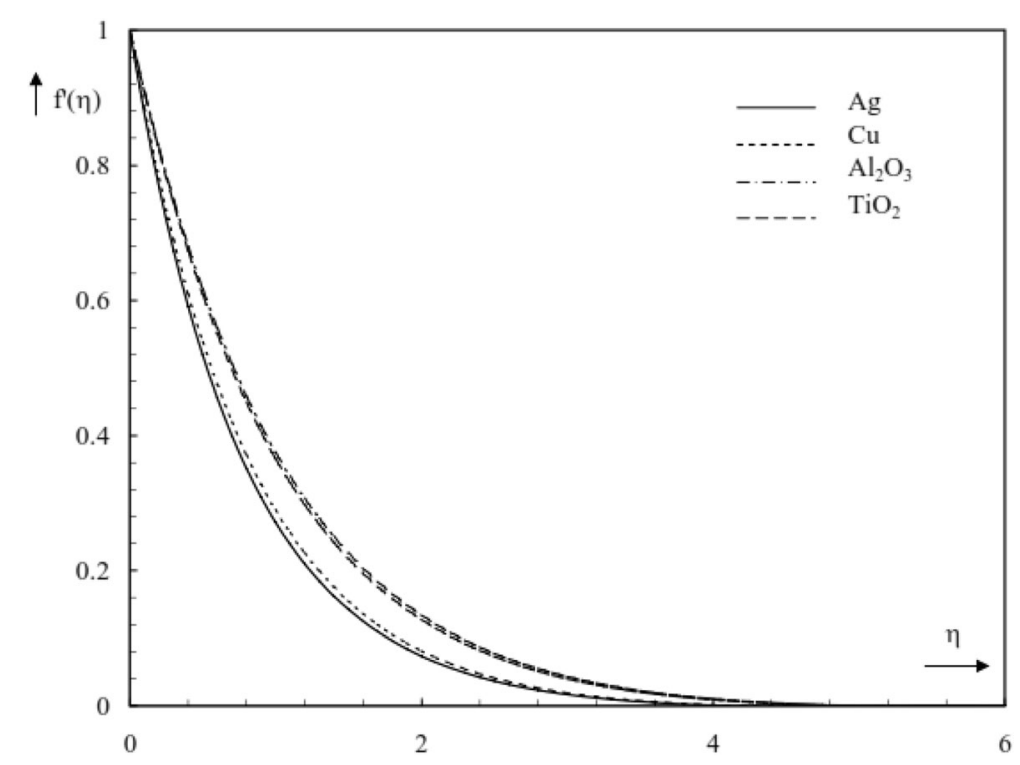

Fig. 3 Velocity profile for different types of nanofluids for $S=M=f_{w}=0.1$ and $A=B=\lambda=0.5$ 


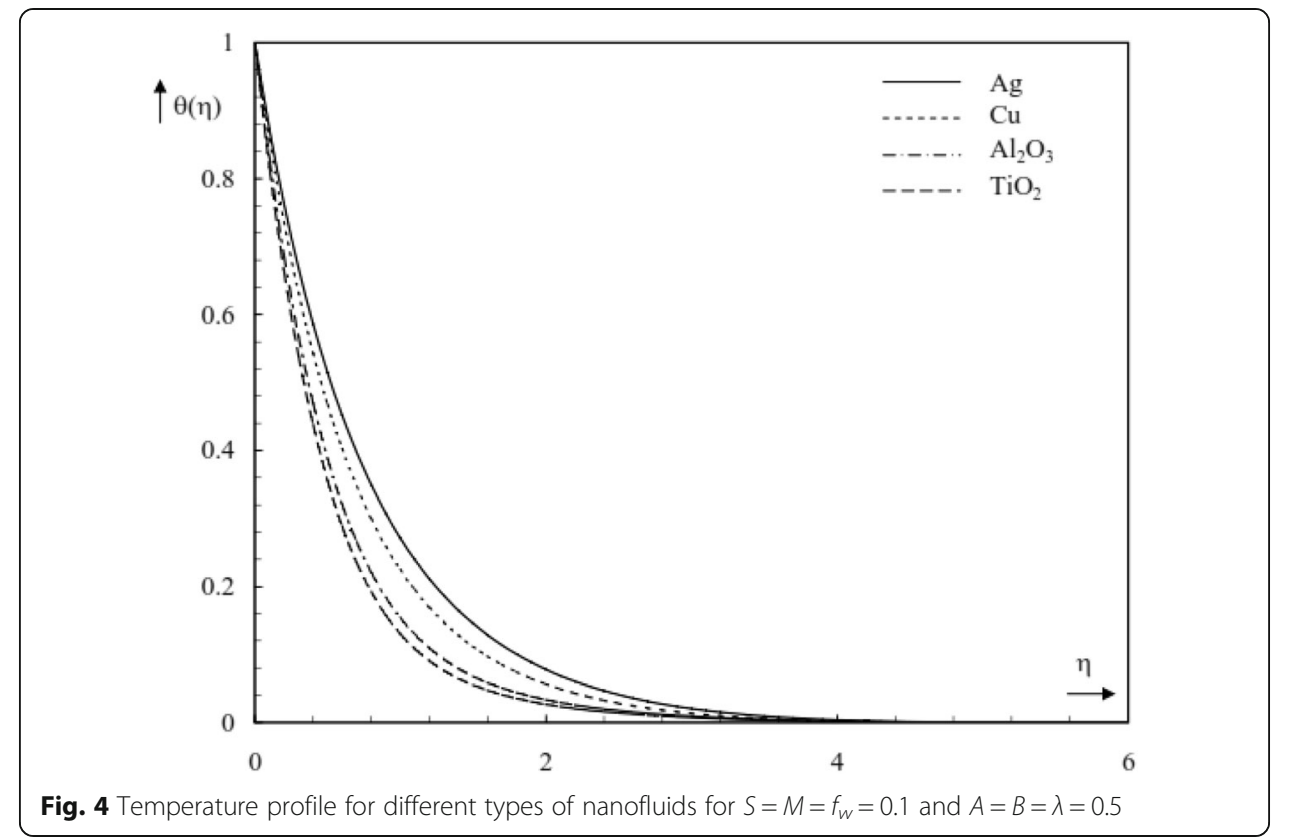

In view of the relations (5)-(10), the Eqs. (2) and (3) become

$$
\begin{aligned}
& f^{\prime \prime \prime}+(1-\phi)^{2.5}\left\{D_{1}\left[f f^{\prime \prime}-\left(f^{\prime}\right)^{2}-S\left(f^{\prime}+\frac{\eta}{2} f^{\prime \prime}\right)\right]+\left(D_{2} \lambda \theta-M \sin ^{2} \gamma f^{\prime}\right)\right\}=0, \\
& \theta^{\prime \prime}+(1-\phi)^{2.5} D_{1}\left(A f^{\prime}+B \theta\right)+\frac{\operatorname{Pr} D_{2}}{D_{3}}\left\{f \theta^{\prime}-f^{\prime} \theta-A\left[\frac{\eta \theta^{\prime}}{2}+2 \theta\right]\right\}=0 .
\end{aligned}
$$

With boundary conditions

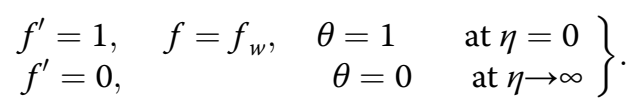

Here, the prime denotes differentiation with respect to $\eta, f$ is the similarity function, $\theta$ is the dimensionless temperature, $\operatorname{Pr}=\frac{\left(\rho C_{P}\right)_{f} v_{f}}{k_{f}}$ is the Prandtl number, $M=\frac{\sigma_{n f} B_{0}^{2}}{a \rho_{f}}$ is the magnetic parameter, $S=c / a$ is the unsteadiness parameter, and $\lambda=\frac{g(\rho \beta)_{f}}{\rho_{f}} \frac{b}{a^{2}}$ is the buoyancy or convection parameter. Also, $\lambda>0$ and $\lambda<0$ correspond to assisting and opposing flows respectively while $\lambda=0$ is for forced convection flow situation. Furthermore, $D_{1}, D_{2}$, and $D_{3}$ are constants given by

$$
D_{1}=(1-\phi)+\frac{\rho_{P}}{\rho_{f}} \phi, \quad D_{2}=(1-\phi)+\frac{\left(\rho C_{P}\right)_{P}}{\left(\rho C_{P}\right)_{f}} \phi, \quad D_{3}=\frac{k_{n f}}{k_{f}} .
$$

When $A=B=M=f_{w}=\phi=0$, the present problem reduces to Ishak et al. [37]. The quantities of physical interest in this problem are the wall shear stress and local rate of heat transfer which are respectively measured in terms of the local skin friction coefficient $\left(C f_{x}\right)$ and the local Nusselt number $\left(N u_{x}\right)$ which are defined by

$$
\frac{1}{2} R e_{x}^{1 / 2} C f_{x}=\frac{1}{(1-\phi)^{2.5}} f^{\prime \prime}(0)
$$




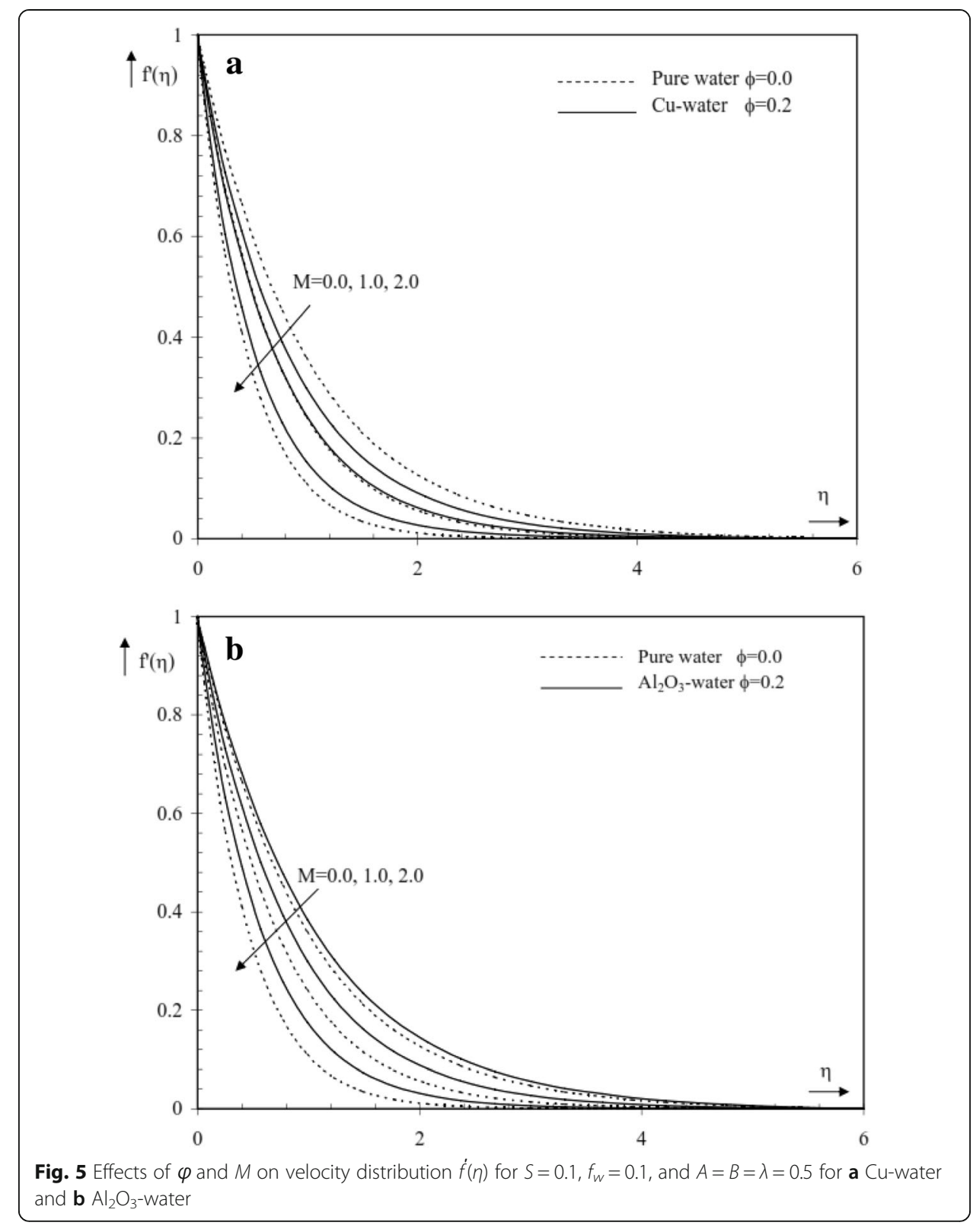

$$
\frac{N u_{x}}{R e_{x}^{1 / 2}}=-D_{3} \theta^{\prime}(0)
$$

where $R e_{x}=\frac{x U_{w}}{v_{f}}$ is the local Reynold number.

\section{Numerical procedure}

The unsteady two-dimensional nanofluid flow over a vertical stretching permeable surface in the presence of an inclined magnetic field and non-uniform heat source/sink is formulated. The Newton Raphson shooting method in conjunction with the fourth-order Runge-Kutta algorithm is used to solve the system of coupled nonlinear ordinary differential Eqs. (11) and (12) with boundary conditions (13). The computer program of the algorithm is executed in MATHEMATICA running on a PC. Firstly, 


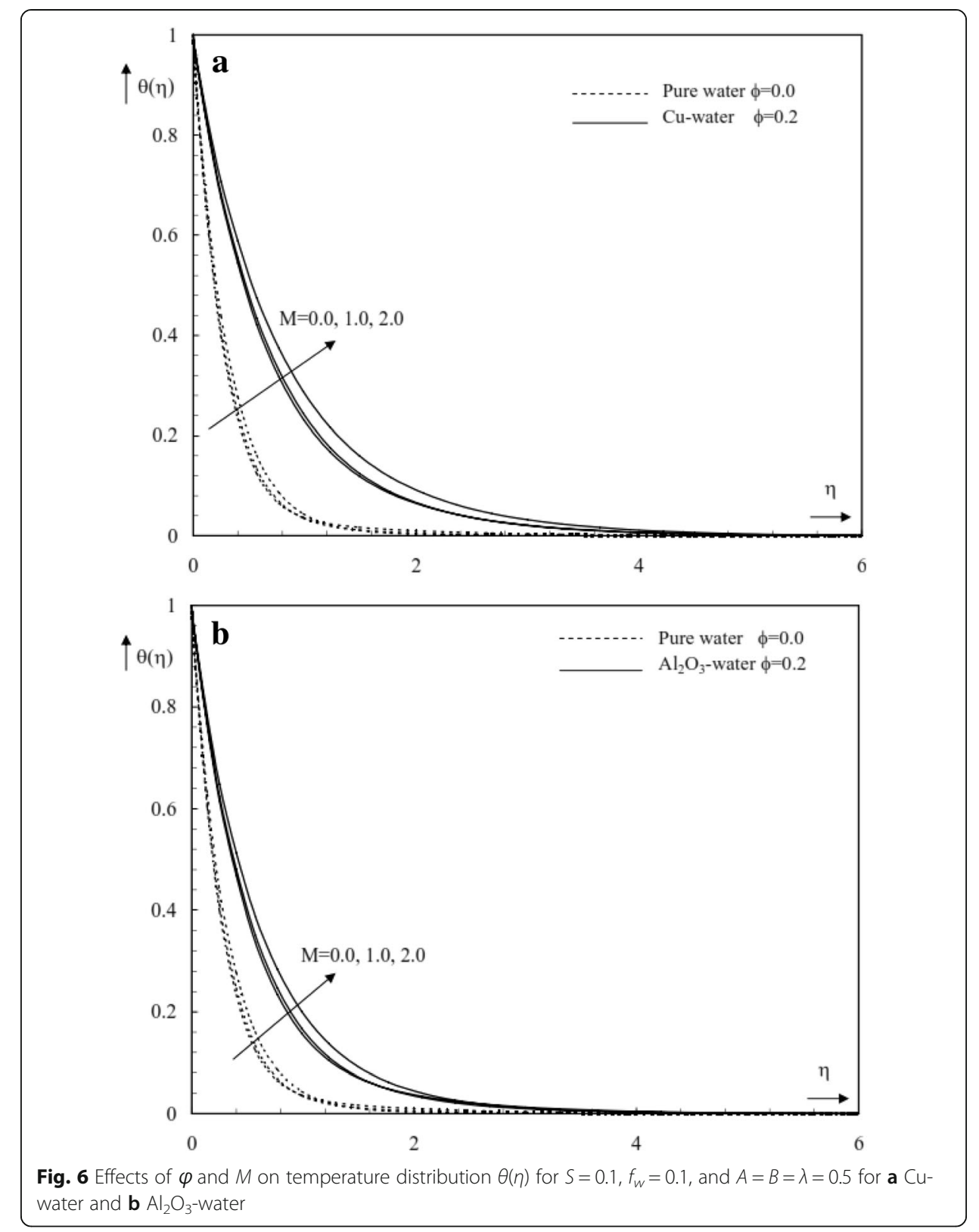

the boundary value problem (BVP) regarding the physical model is converted into the initial value problem (IVP). Since the constructed system (11-12) has one third-order and other two-order equations, so its solution can be attained unless five initial conditions are given. But in Eq. (13), only three initial conditions are defined. Thus, before starting the solution process, suitable initial approximations for $\dot{f}(\infty)=0$ and $\theta(\infty)=0$ are selected, i.e., the boundary conditions (13) are replaced with $\mathrm{f}^{\prime \prime}(0)=\omega_{1}$ and $\theta^{\prime}(0)$ $=\omega_{2}$ (guesses). Also, the upper limit of the independent variable $\eta_{\infty}$ should be chosen finite. Secondly, the Runge-Kutta method is applied and the solution is calculated. Finally, the computed solution will converge if boundary residuals (the difference between the given and the computed values of $\dot{f}(\infty)=0$ and $\theta(\infty)=0$ ) is less than the error tolerance $\left(10^{-6}\right)$. If the computed solution does not meet the convergence 

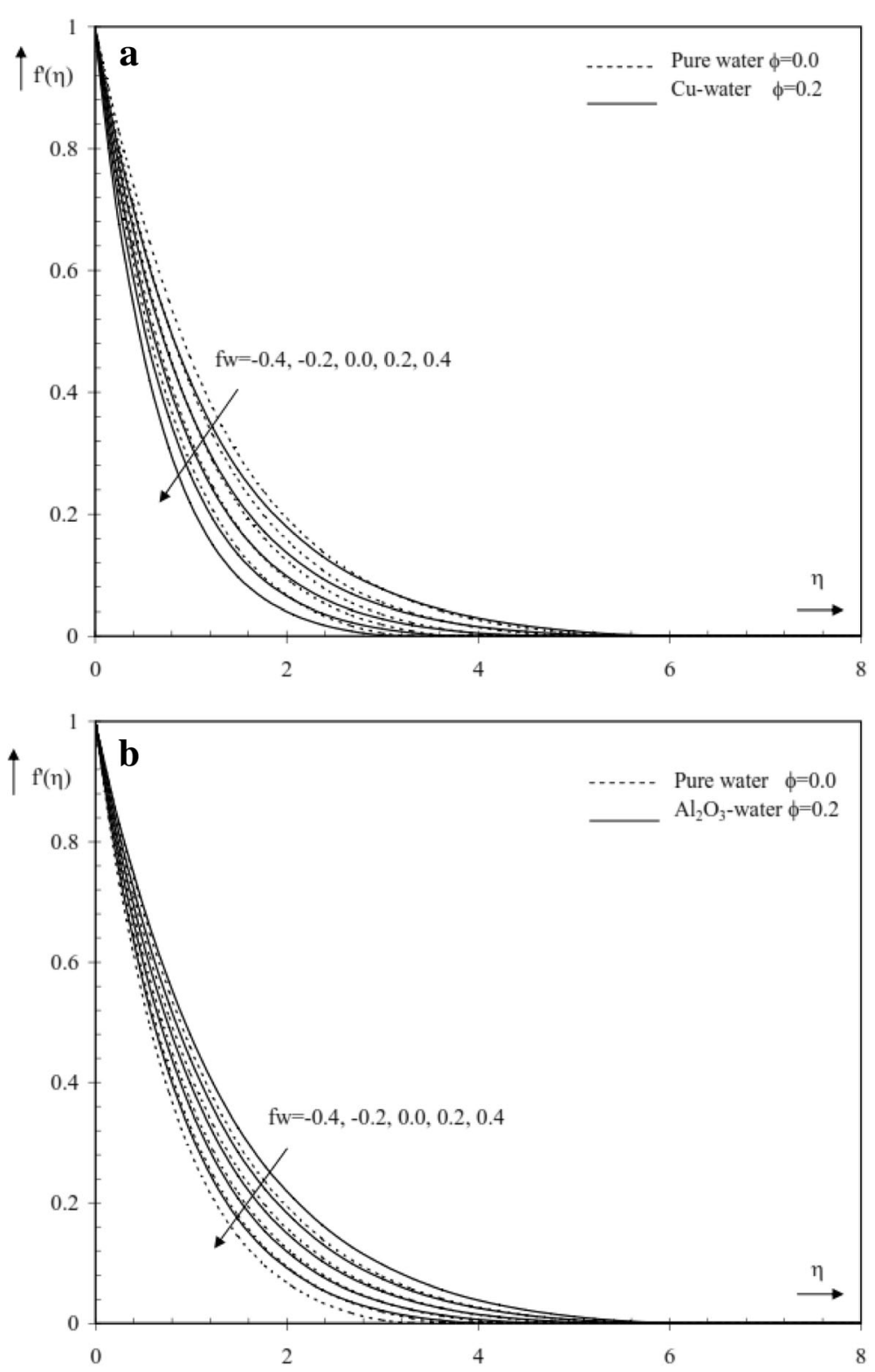

Fig. 7 Effects of $\varphi$ and $f_{w}$ on velocity distribution $f^{\prime}(\eta)$ for $S=M=0.1$ and $A=B=\lambda=0.5$ for a Cu-water and b $\mathrm{Al}_{2} \mathrm{O}_{3}$-water

criterion, then initial guesses are modified by Newton's method. The procedure is continued unless a solution meets the criterion as shown in Fig. 2.

\section{Results and discussions}

The nonlinear ordinary differential Eqs. (11) and (12) subject to the boundary conditions (13) are solved numerically by using the Runge-Kutta algorithm supported by the shooting method with the help of the symbolic computational software MATHEMATICA. In order to assess the accuracy of the numerical method, we have compared our $-\theta^{\prime}(0)$ results with the previously published data of Ishak at el. [37], Grubka and Bobba [38], and Ali [39] for different values of $S, \lambda$, and $\operatorname{Pr}$ at $A=B=M=f_{w}=\phi$ (pure fluid) $=$ 0 . The comparison is listed in Table 2 and found in excellent agreement. Extensive 
numerical computations were carried out for the influence of the emerging non-dimensional governing parameters on the nanofluid velocity and temperature distributions in a wide range of $\pi / 4 \leq \gamma \leq \pi / 2, M \leq 10, \quad 0 \leq S \leq 2, \quad-0.5 \leq A, B, \lambda \leq 0.5$, $0.4 \leq f_{w} \leq 0.4,0 \leq \phi \leq 0.2$, and $\operatorname{Pr}=6.785$. The wall shear stress and local rate of heat transfer are measured in terms of the local skin friction coefficients and the local Nusselt number. The numerical results are presented in Tables 3, 4, and 5 and graphically in Figs. 3, 4, 5, 6, 7, 8, 9, 10, 11, 12, 13, 14, 15, 16, 17, 18, and 19, for four different types of nanoparticles, namely silver $\mathrm{Ag}$, copper $\mathrm{Cu}$, alumina $\mathrm{Al}_{2} \mathrm{O}_{3}$, and titania $\mathrm{TiO}_{2}$, by using water as a base fluid with the pertinent parameters.

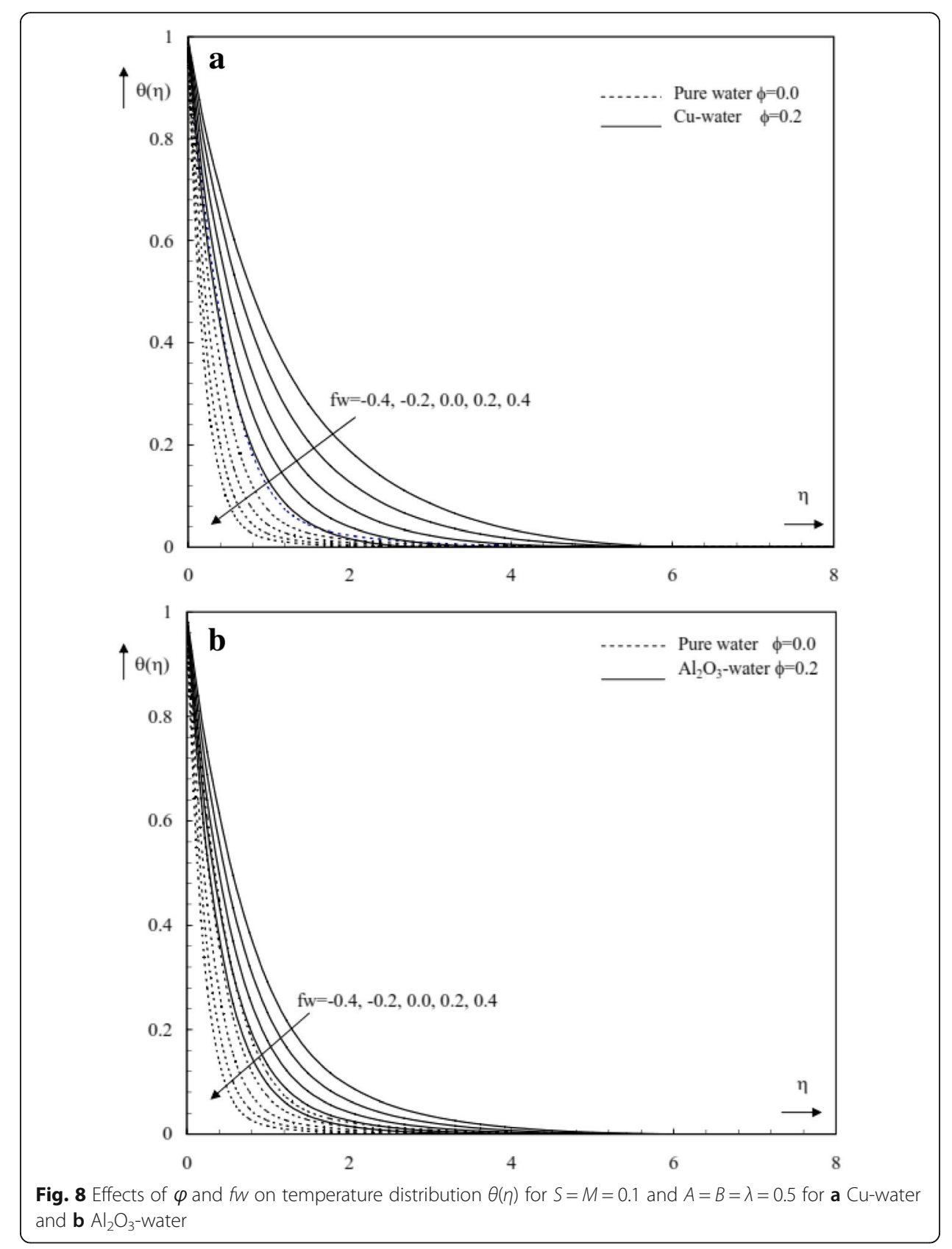



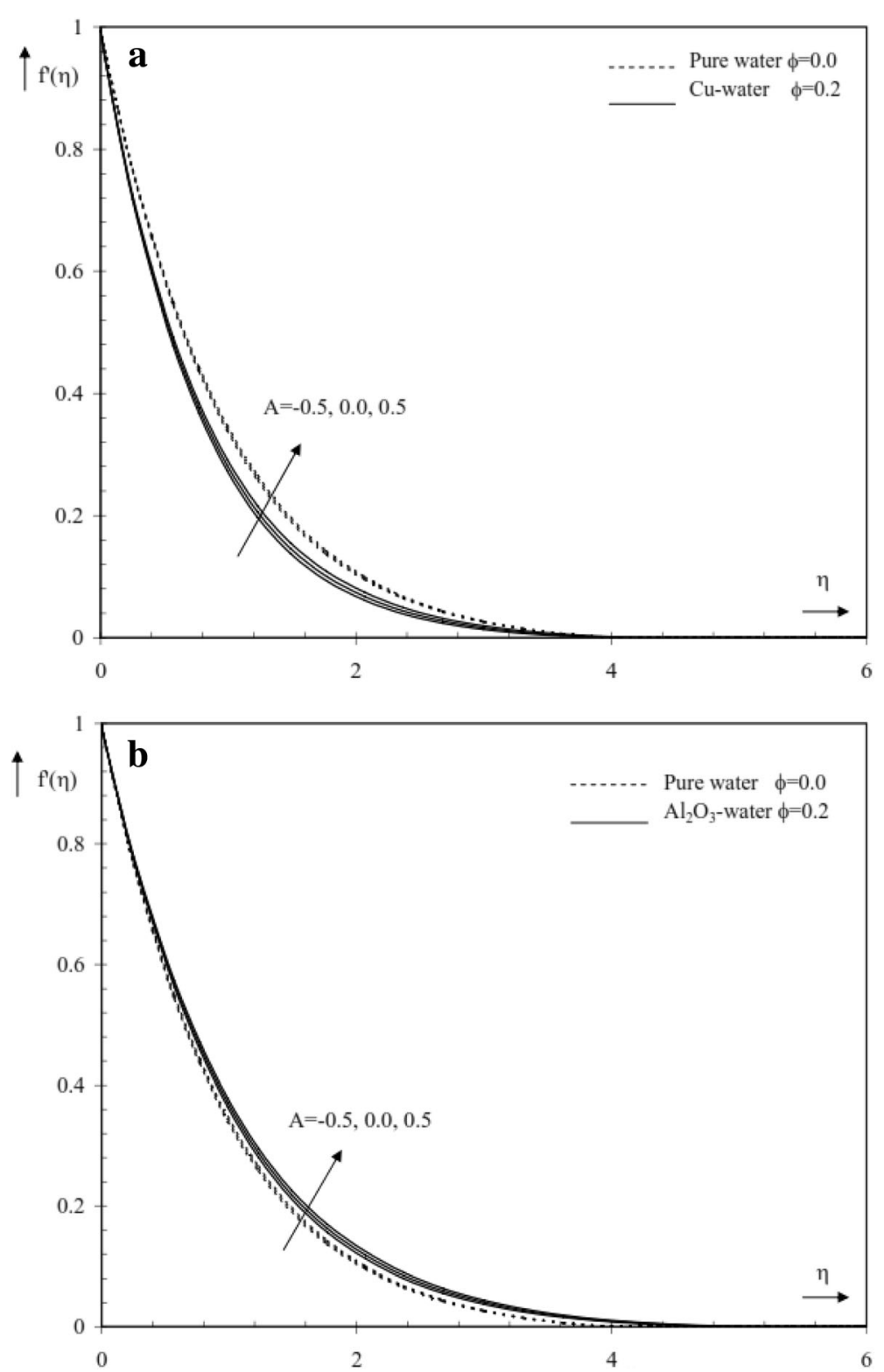

Fig. 9 Effects of $\varphi$ and $A$ on velocity distribution $f^{\prime}(\eta)$ for $S=M=f_{w}=0.1$ and $B=\lambda=0.5$ for a Cu-water and b $\mathrm{Al}_{2} \mathrm{O}_{3}$-water

From Table 3, as compared to the case of no heat source/sink $(A=B=0)$, the skin friction coefficient and the local Nusselt number are augmented due to heat $\operatorname{sink}(A<0$ and $B<0)$ whereas the opposite results hold with heat source $(A>0$ and $B>0)$, for all values of nanoparticle volume fraction. On the other hand, the skin friction coefficient increases for $\mathrm{Ag}$-water and $\mathrm{Cu}$-water by increasing nanoparticle volume fraction $\phi$, whereas the opposite results occur for $\mathrm{Al}_{2} \mathrm{O}_{3}$-water and $\mathrm{TiO}_{2}$-water. Additionally, the local Nusselt number decreases by increasing nanoparticle volume fraction $\phi$ for all values of heat source/sink $A$ and $B$. From Table 4, it is observed that the skin friction coefficient and the local Nusselt number increases by increasing $f_{w}$ (for pure fluid ( $\phi=$ 0 ) and four different types of nanofluids). These results are similar to that reported by Das [44]. On the other hand, the skin friction coefficient increases by increasing 


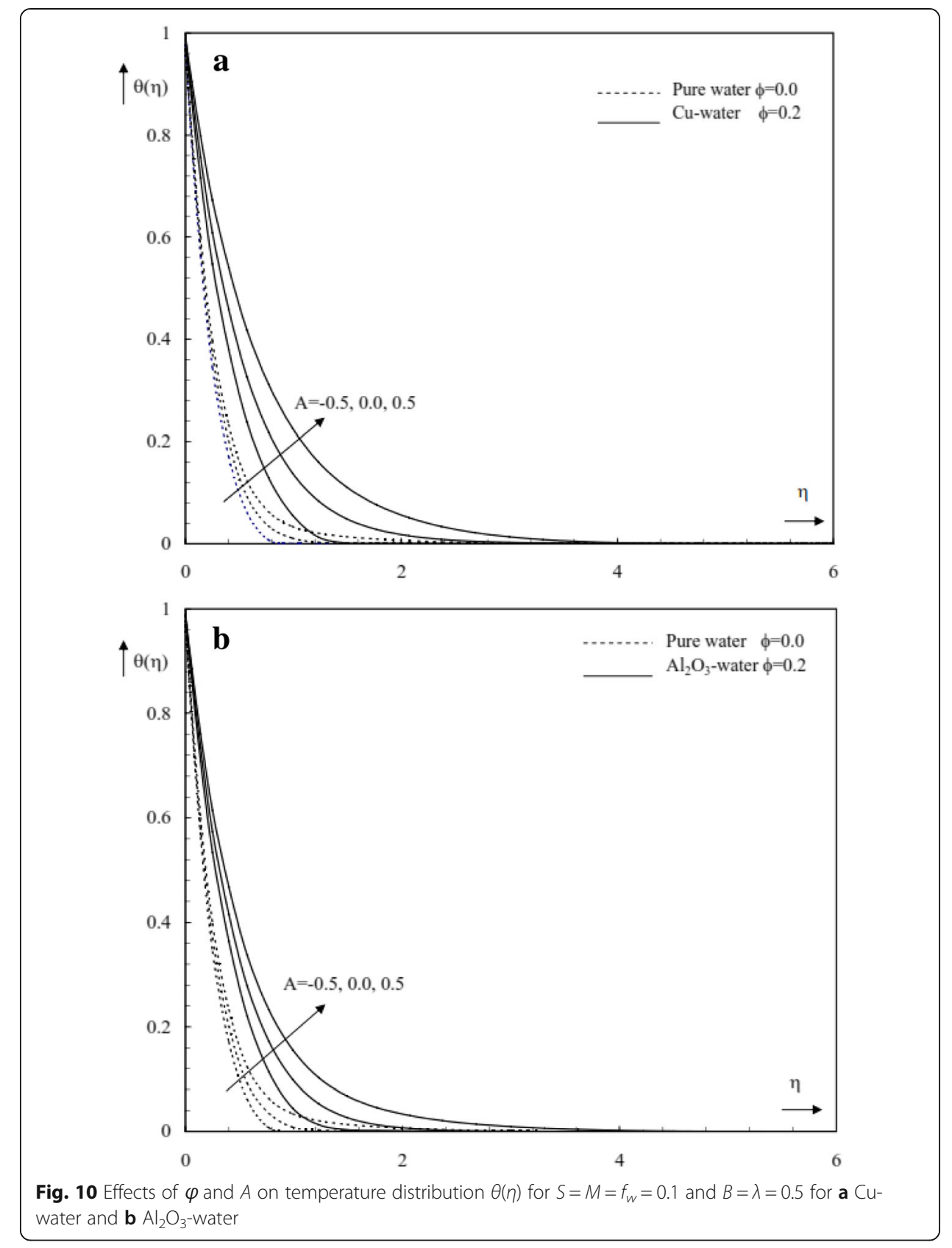

magnetic parameter $M$ for both cases of pure fluid $(\phi=0)$ and nanofluids. Also, for both cases of pure and nanofluid, the local Nusselt number increases by increasing the magnetic parameter $M$ for the suction case $\left(f_{w}>0\right)$, whereas the opposite results occur for the injection case $\left(f_{w}<0\right)$. Furthermore, the local Nusselt number in nanofluids is lower than in a pure fluid. This means that the nanofluid type will be important in the cooling and heating processes. In addition, from Table 5, it is observed that the skin friction coefficient and the local Nusselt number increase by increasing unsteadiness parameter $S$, for both cases of pure and nanofluids. On the other hand, the skin friction coefficient decreases by increasing convection parameter $\lambda$ whereas the opposite results hold with the local Nusselt number for both cases of pure and nanofluids. Additionally, 

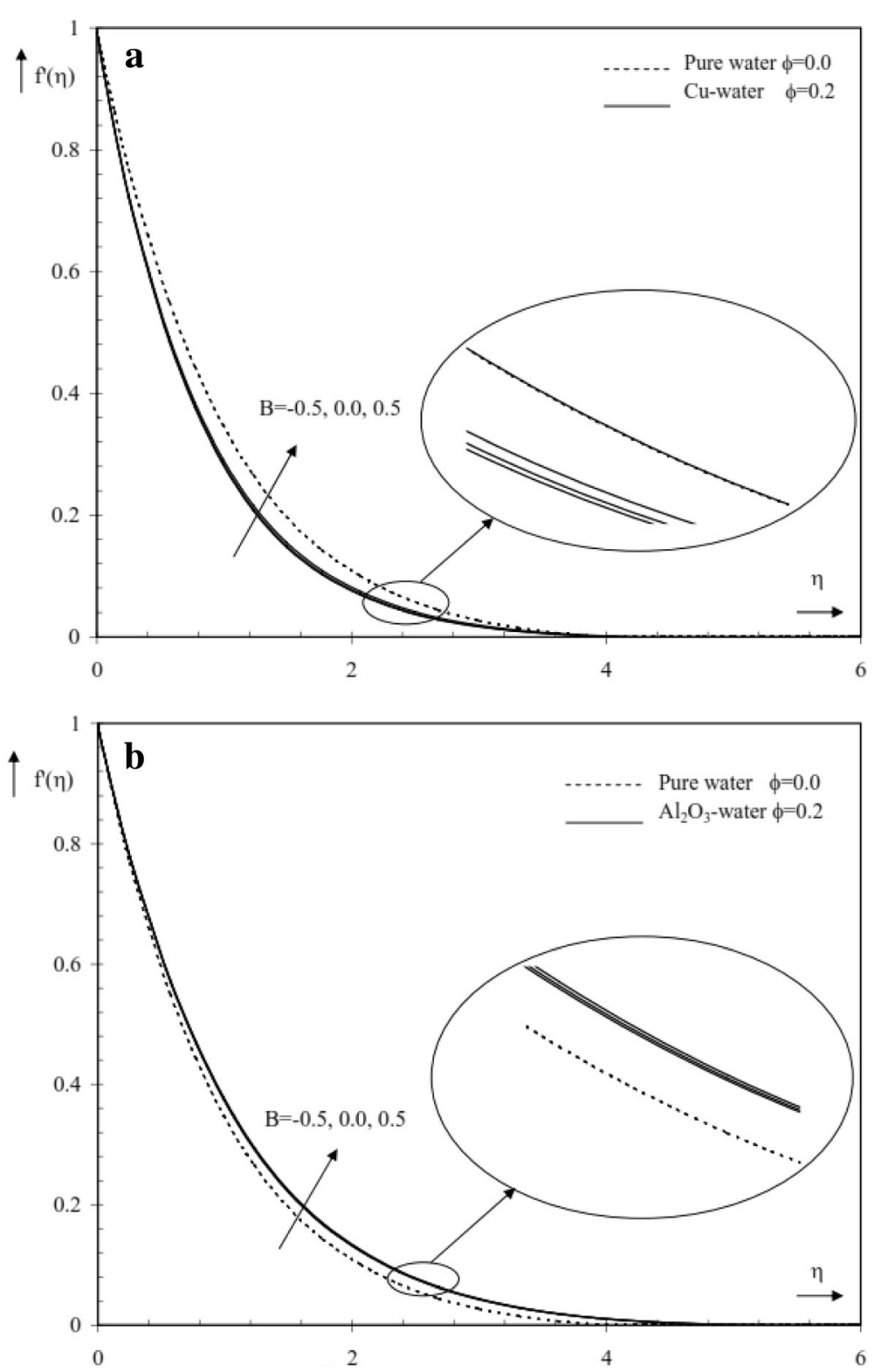

Fig. 11 Effects of $\varphi$ and $B$ on velocity distribution $f^{\prime}(\eta)$ for $S=M=f_{w}=0.1$ and $A=\lambda=0.5$ for a Cu-water and $\mathbf{b} \mathrm{Al}_{2} \mathrm{O}_{3}$-water

the skin friction coefficient for $\mathrm{Cu}$-water and $\mathrm{Ag}$-water is higher than in a pure fluid whereas the opposite results occur for $\mathrm{Al}_{2} \mathrm{O}_{3}$-water and $\mathrm{TiO}_{2}$-water.

Figures 3 and 4 display the behavior of the velocity and temperature for different types of nanofluids. It is observed that both momentum and thermal boundary layer thickness change with the change in the type of nanoparticles. Figure 4 displays that the minimum value of the temperature is obtained by adding titanium oxide to the fluid, while by choosing silver as the nanoparticle, maximum value of the temperature is observed, i.e., the thermal boundary layer thickness is related to the increased thermal conductivity of different types of nanofluids. In fact, as seen in Table 1, the low value of thermal diffusivity causes a drop in the thermal boundary layer thickness as plotted in Fig. 4. Furthermore, the velocity and temperature profiles start with the plate 


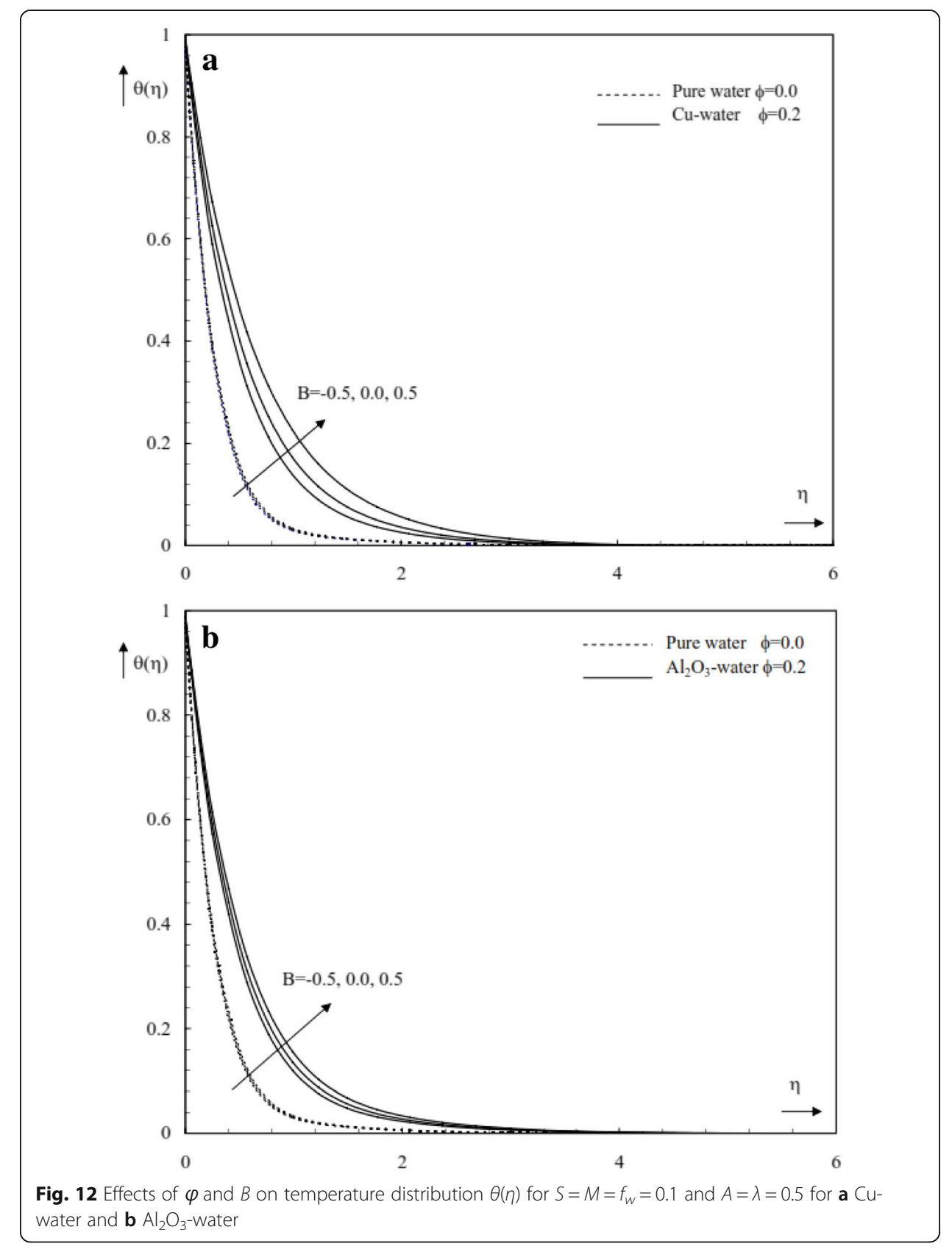

velocity and then decrease monotonically to zero, satisfying the far field boundary condition. Figures 5, 6, 7, 8, 9, 10, 11, 12, 13, 14, 15, and 16 are drawn (at $\operatorname{Pr}=6.785$ and $\gamma$ $=\pi / 3$ ) in order to study the influence of magnetic, suction/injection, convection, heat source/sink, and unsteadiness parameters on the velocity and temperature fields for two selected different water-based nanofluids $\mathrm{Cu}$-water and $\mathrm{Al}_{2} \mathrm{O}_{3}$-water. Figures 5 and 6 show the effects of magnetic parameter $M$ on velocity profiles and temperature distribution: (a) pure and copper-water nanofluids and (b) pure and alumina-water nanofluids. It is observed that the velocity profile in the boundary layer decreases by increasing the magnetic parameter whereas the reverse trend is observed for the temperature profile. In other words, the application of a transverse magnetic field to an 

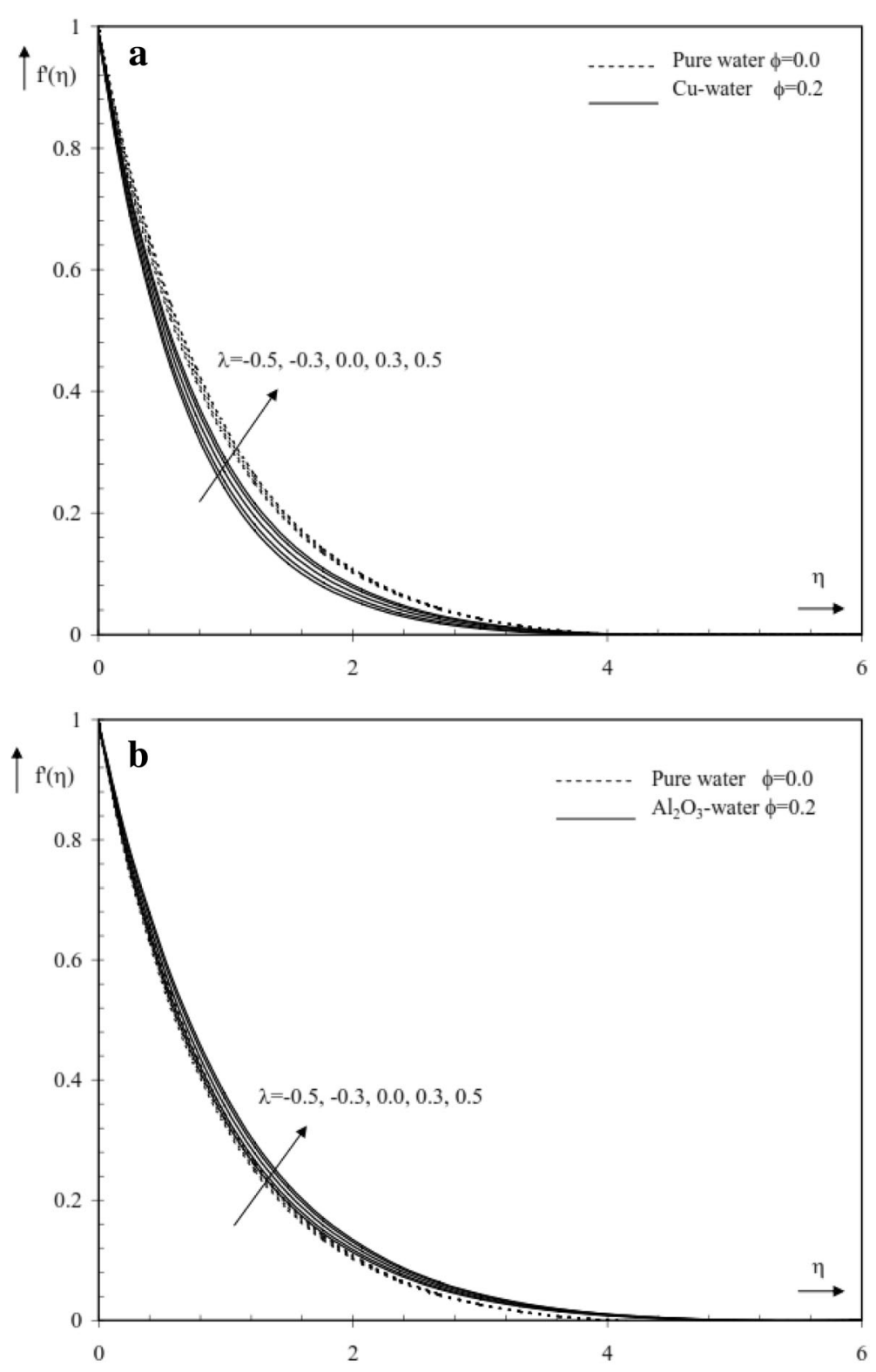

Fig. 13 Effects of $\varphi$ and $\lambda$ on velocity distribution $\dot{f}(\eta)$ for $S=M=f_{w}=0.1$ and $A=B=0.5$ for a Cu-water and $\mathbf{b ~} \mathrm{Al}_{2} \mathrm{O}_{3}$-water

electrically conducting fluid gives rise a resistive type force called the Lorentz force. This force has the tendency to slow down the motion of the fluid in the boundary layer and to increase its temperature (as shown in Figs. 5 and 6). Also, the temperature of the $\mathrm{Cu}$-water and $\mathrm{Al}_{2} \mathrm{O}_{3}$-water nanofluids is increased significantly as compared to that of the pure fluid by increasing the magnetic parameter. Furthermore, it is worth mentioning here that in Fig. 6 the thermal boundary layer thickness for $\mathrm{Cu}$-water nanofluid is generally greater than the $\mathrm{Al}_{2} \mathrm{O}_{3}$-water nanofluid and is attributable to the greater thermal conductivity of copper compared with alumina. Figures 7 and 8 depict the influences of suction/injection parameters $f_{w}$ on velocity profiles and temperature distributions, for both cases of pure and nanofluids. It is observed that the velocity and temperature profiles decrease by increasing the suction parameter $\left(f_{w}>0\right)$ whereas an 

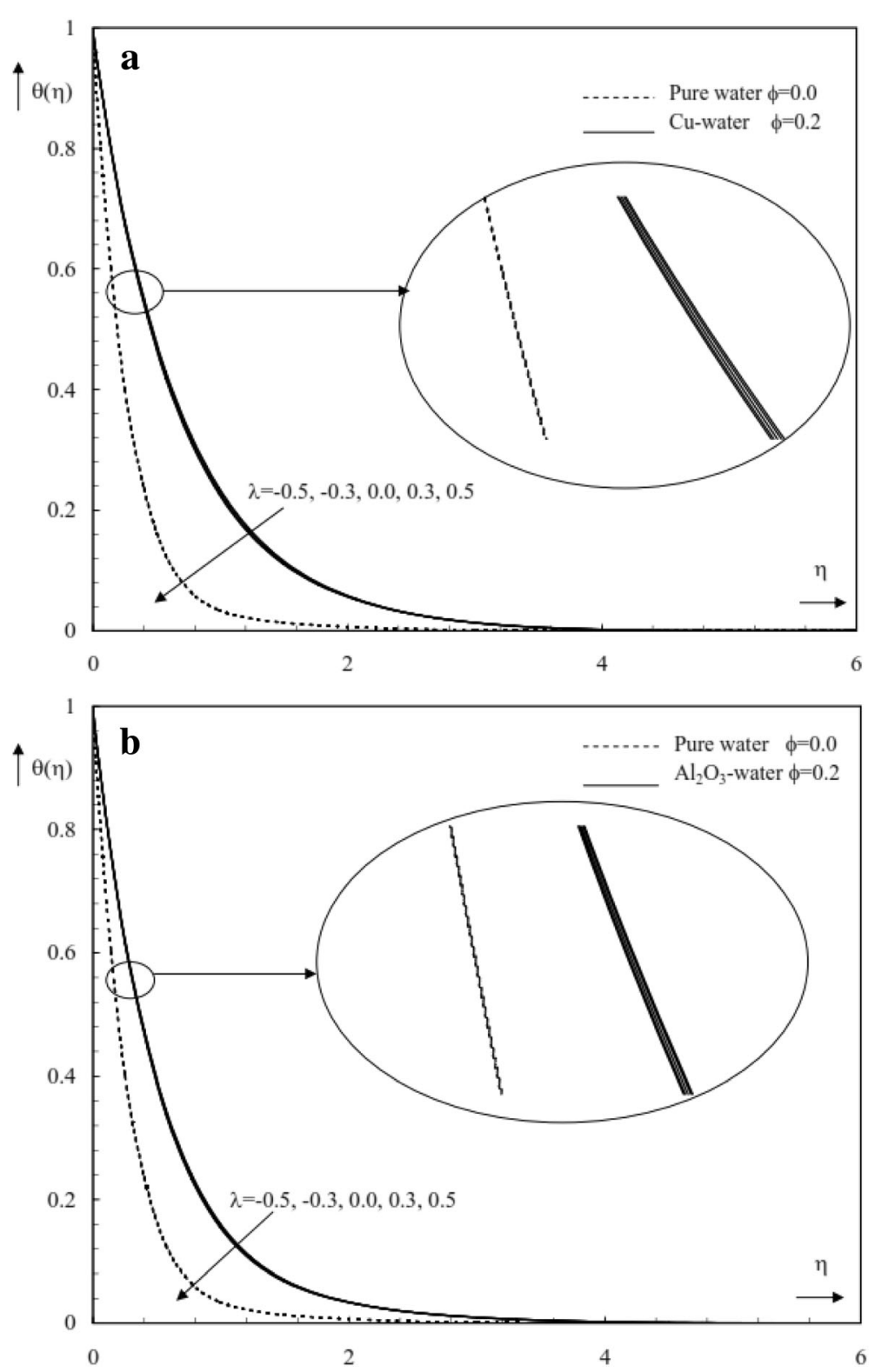

Fig. 14 Effects of $\varphi$ and $\lambda$ on temperature distribution $\theta(\eta)$ for $S=M=f_{w}=0.1$ and $A=B=0.5$ for a Cuwater and $\mathbf{b} \mathrm{Al}_{2} \mathrm{O}_{3}$-water

opposite effect is observed for the injection parameter $\left(f_{w}<0\right)$. Furthermore, the increment in the effect of $f_{w}$ on the thermal boundary layer thickness is partially more in $\mathrm{Cu}$-water nanofluid case than in $\mathrm{Al}_{2} \mathrm{O}_{3}$-water nanofluid case as shown in Fig. 8. Figures $9,10,11$, and 12 show the response in nanofluid velocity and temperature distributions across the boundary layer to various values of heat generation source $(A>0$ and $B>0)$ and heat absorption sink $(A<0$ and $B<0)$. The heat source leads to increase velocity profile and boundary layer thickness, whereas the opposite results occur with heat sink as shown in Figs. 9 and 11. The velocity of the pure fluid is higher than the velocity of $\mathrm{Cu}$-water, whereas the opposite results occur for $\mathrm{Al}_{2} \mathrm{O}_{3}$-water. Additionally, it is clear from Figs. 10 and 12 that the thermal boundary layer decreases for $A<0$ and $B<0$; 


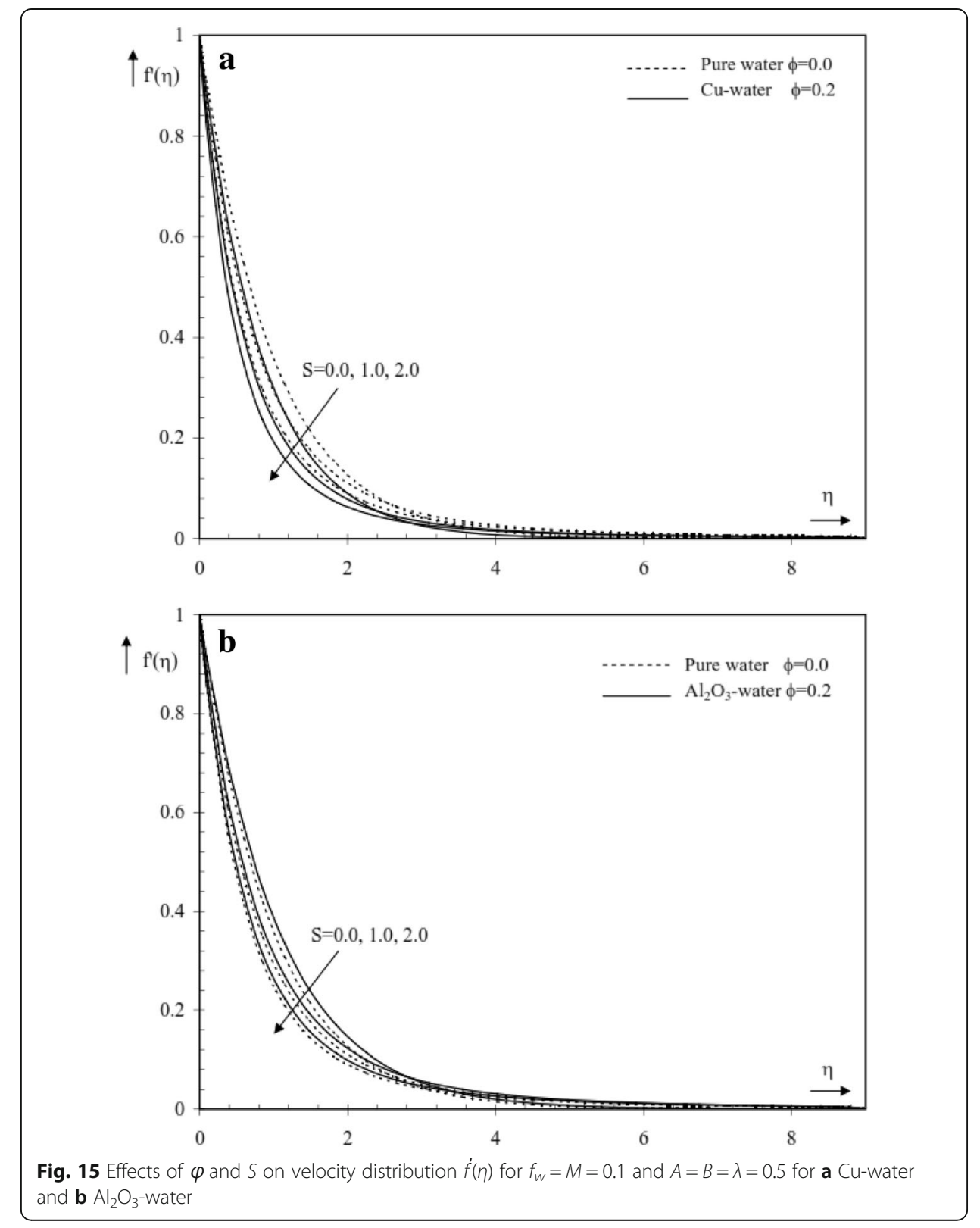

physically, the internal heat sink may be exploited to successfully cool the regime. However, the opposite trend is observed for heat source $A>0$ and $B>0$. Notice that the effects of heat source/sink parameters $(A$ and $B)$ on the velocity and temperature profiles for the nanofluid case are stronger than those of the pure fluid case. Moreover, the effect of parameter $A$ on the velocity and temperature profiles is stronger than that of parameter $B$ for both cases of pure and nanofluids. Figures 13 and 14 show the effects of convection parameter $\lambda$ on the velocity and temperature profiles for both cases of pure and nanofluids. It is observed that the hydrodynamic boundary layer thickness and velocity profile increase by increasing convection parameter $\lambda$ whereas the reverse trend is observed for the temperature profile and thermal boundary layer thickness. Also, the effect of convection parameter on the velocity of the pure fluid is clearer than its effect on the temperature for both cases of pure and nanofluids. Figures 15 and 16 


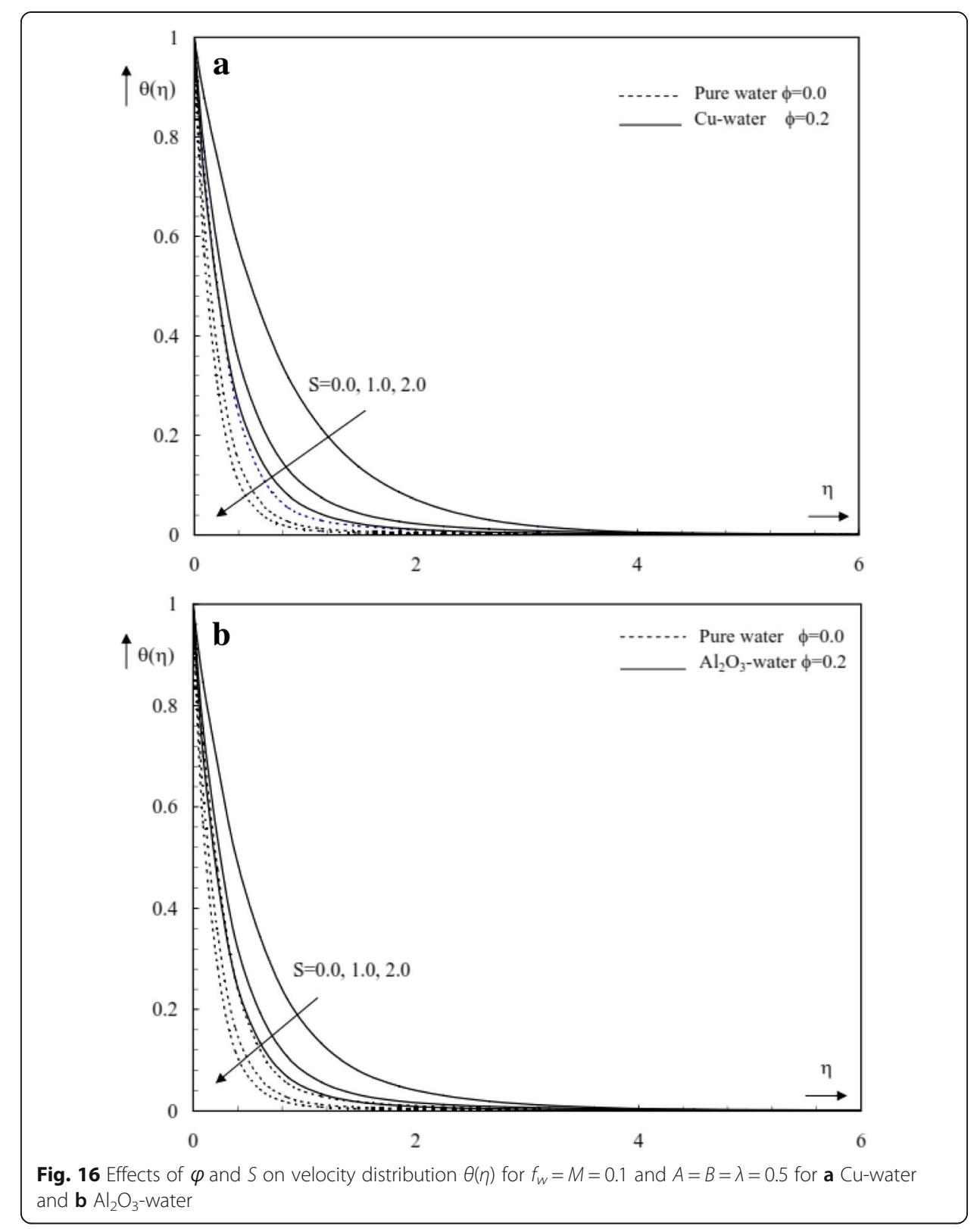

depict the effects of unsteadiness parameter $S$ on the velocity and temperature profiles for both cases of pure and nanofluids. It is observed that the velocity and temperature profiles in the boundary layer decrease by increasing unsteadiness parameter. On the other hand, the temperature of the $\mathrm{Cu}$-water and $\mathrm{Al}_{2} \mathrm{O}_{3}$-water is increased significantly as compared to that of the pure fluid by increasing convection and unsteadiness parameters. These results for pure fluid are similar to that reported by Ishak at el. [37]. Finally, Figs. 17, 18, and 19 depict the influence of the magnetic field inclination angle $\gamma$ at $\operatorname{Pr}=6.785$ on the velocity flow, the skin friction coefficient, and the local Nusselt number for both cases of pure and nanofluids, respectively. Figure $17 \mathrm{a}$ and $\mathrm{b}$ display the effect of the magnetic field inclination angle $\gamma$ on the velocity profiles. It is clear that the increase in the angle of inclination $\gamma$ decreases the velocity profile of the nanofluids. The reason behind this result is the increase in angle of inclination which causes 


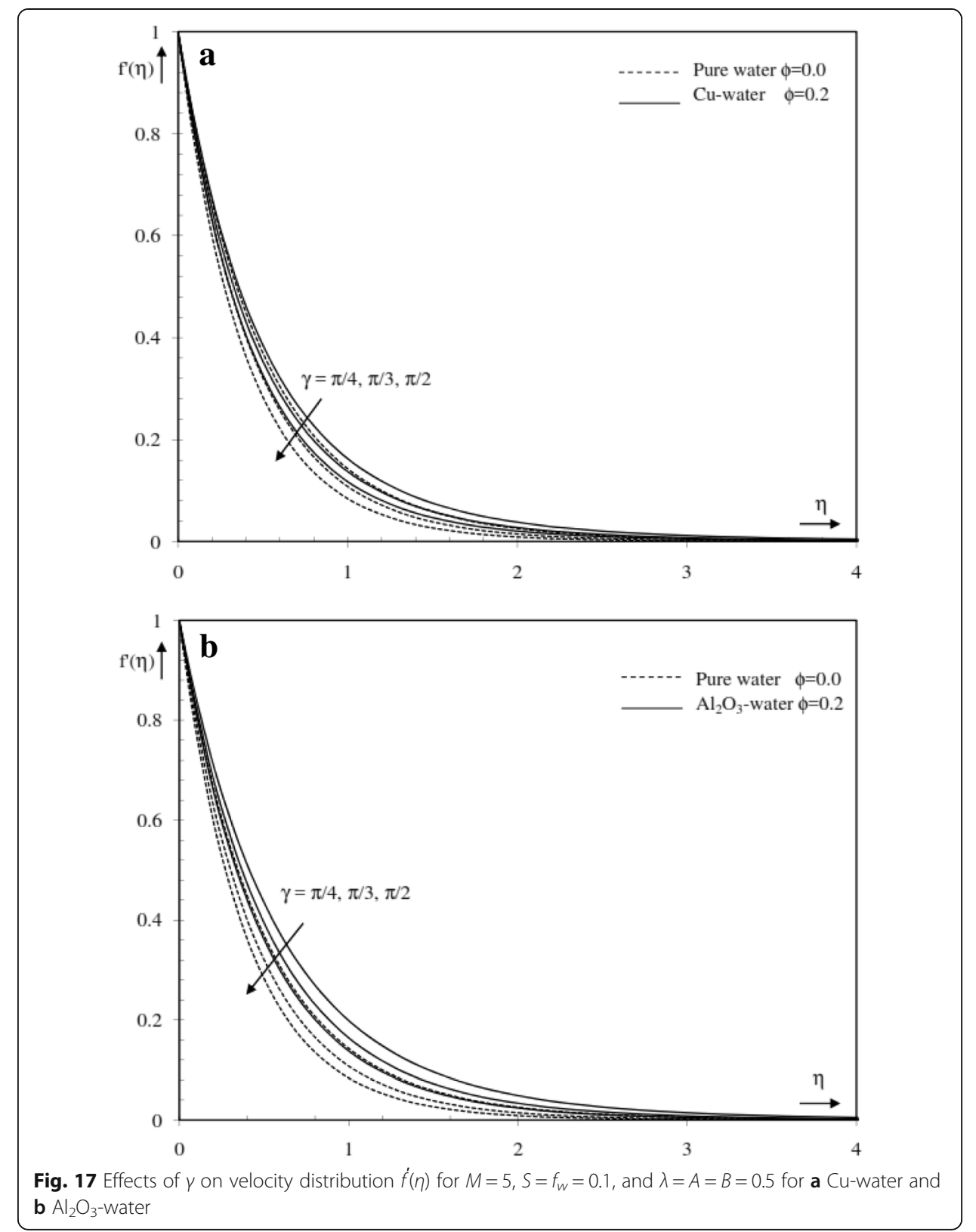

to strengthen the magnetic field. Due to the enhanced magnetic field, it generates the opposite force to the flow (Lorentz force). This force declines the momentum boundary layer thickness. Effects of the magnetic field $M$ and the magnetic field inclination angle $\gamma$ on the skin friction coefficient and the local Nusselt number given by Eqs. (15) and (16) are shown in Figs. 18 and 19. Figures 18 and 19 show that when $\gamma$ increases from $\pi / 4$ to $\pi / 2$, the skin friction coefficient increases for $\mathrm{Cu}$-water, $\mathrm{Al}_{2} \mathrm{O}_{3}$-water, and pure fluid cases whereas the local Nusselt number decreases for nanoparticle fluid. It is seen that when $M$ increases, the skin friction coefficient increases whereas the local Nusselt number decreases. Furthermore, it is evident from Fig. 18 that the increase in magnetic field $M$ and angle of inclination $\gamma$ causes an increase in skin friction coefficient profile and this effect is high on the pure fluid case compared to the nanofluid case. Generally, the increase in the value of the magnetic field and angle of inclination enhances the 


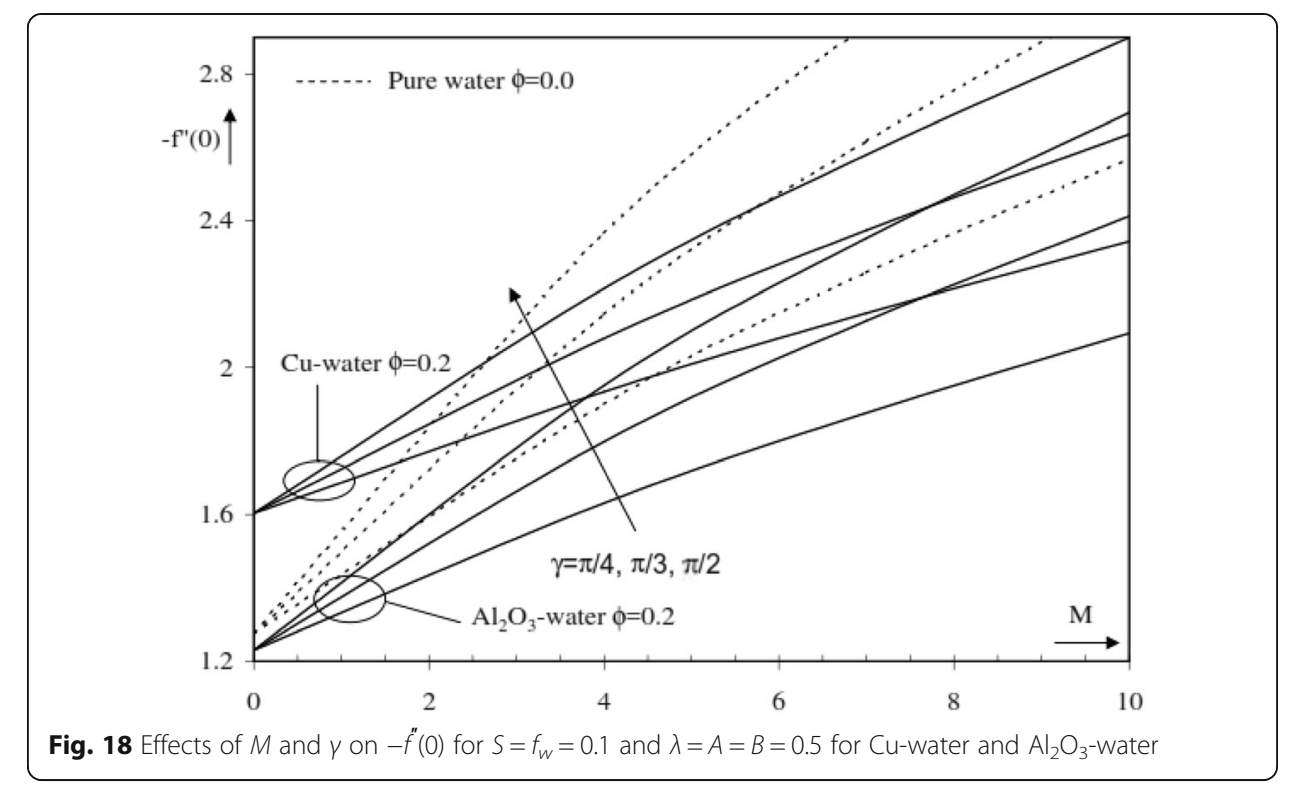

value of skin friction coefficient; however, it directly helps to reduce the value of local Nusselt number. These results are similar to that obtained by Raju at el. [40].

\section{Conclusions}

In the present paper, we have studied numerically as well as physically the nanofluid flow over a permeable unsteady stretching surface with non-uniform heat source/sink in the presence of an inclined magnetic field. The shooting method is employed to solve the nonlinear system of equations arising in this particular problem. The variations of different parameters have been investigated. The results are discussed through graphs and tabular form. Comparisons with existing results are presented.

Some conclusions obtained from this investigation are summarized as follows:

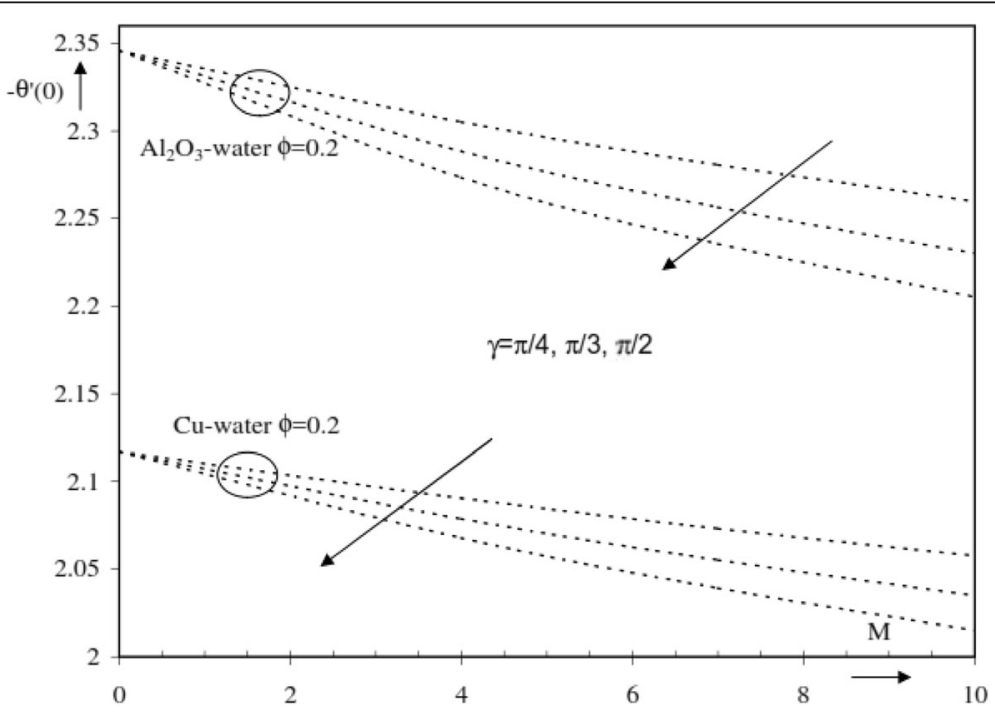

Fig. 19 Effects of $M$ and $\gamma$ on $-\theta^{\prime}(0)$ for $S=f w=0.1$ and $\lambda=A=B=0.5$ for Cu-water and $\mathrm{Al}_{2} \mathrm{O}_{3}{ }^{-}$water 
1. Inclined angle strengthens the magnetic field, it has the capability to reduce the velocity profile of the flow and the local Nusselt number, and it improves the skin friction coefficient.

2. At $\gamma=\pi / 2$, the angle of inclination of the magnetic field acts like a transverse magnetic field.

3. It is found that by using various types of nanofluids, the velocity and temperature change, which means that the nanofluids in the presence of a magnetic field and heat source/sink are important in the cooling and heating processes.

4. The heat transfer rate increases with heat $\operatorname{sink} A<0$ and $B<0$, whereas the opposite results hold with heat source $A>0$ and $B>0$ for all different types of nanofluid.

5. Increasing suction parameter $\left(f_{w}>0\right)$ leads to skin friction coefficient and local Nusselt number increases, whereas the opposite results hold with the injection parameter $\left(f_{w}<0\right)$ for both cases of pure fluid and nanofluids.

6. Increasing unsteadiness parameter $S$ leads to the skin friction coefficient and the local Nusselt number increase for both cases of pure fluid and nanofluids.

7. Increasing convection parameter $\lambda$ leads to the skin friction coefficient decrease, whereas the opposite results hold with the local Nusselt number for both cases of pure fluid and nanofluid.

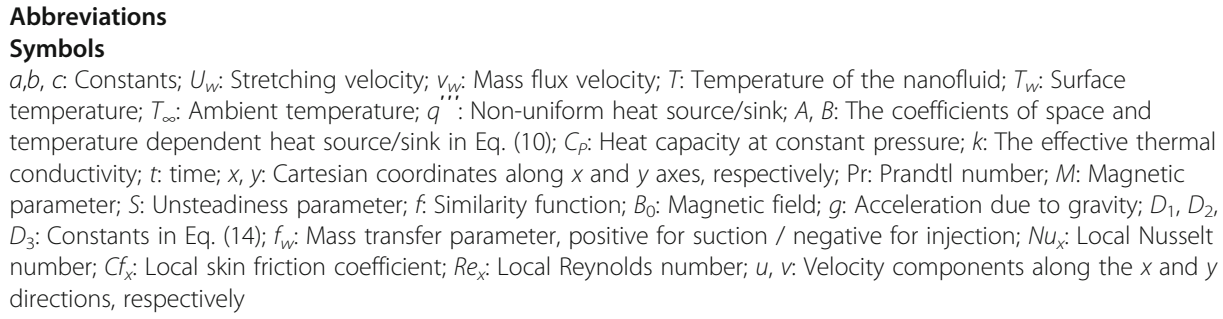

Authors' contributions

NSE analyzed and interpreted the data, performed the numerical solution for the governing partial differential equations of the present problem, wrote the manuscript, and read and approved the final manuscript. 


\section{Publisher's Note}

Springer Nature remains neutral with regard to jurisdictional claims in published maps and institutional affiliations.

\section{Received: 14 June 2018 Accepted: 26 October 2018}

Published online: 02 May 2019

\section{References}

1. Sakiadis, B.C.: Boundary layer behavior on continuous solid surfaces. AICHE J. 7, 26-28 (1961)

2. Erickson, L.E., Fan, L.T., Fox, V.G.: Heat and mass transfer on a continuous flat plate with suction/injection. Ind. Eng. Chem. Fundam. 5, 19-25 (1966)

3. Tsou, F.K., Sparrow, E.M., Goldstein, R.J.: Flow and heat transfer in the boundary layer on a continuous moving surface. Int. J. Heat Mass Transf. 10, 219-235 (1967)

4. Crane, L.J.: Flow past a stretching plate. Z. Angew. Math. Phys. 21, 645-647 (1970)

5. Hayat, T., Saif, S., Abbas, Z.: The influence of heat transfer in an MHD second grade fluid film over an unsteady stretching sheet. Phys. Letters A. 372, 5037-5045 (2008)

6. Prasad, K.V., Vajravelu, K.: Heat transfer in the MHD flow of a power law fluid over a non-isothermal stretching sheet. Int. J. Heat Mass Transf. 52, 4956-4965 (2009)

7. Kumaran, V., Vanav Kumar, A., Pop, I.: Transition of MHD boundary layer flow past a stretching sheet. Commun. Nonlinear Sci. Nume. Simul. 15, 300-311 (2010)

8. Mukhopadhyay, S.: Heat transfer analysis for unsteady MHD flow past a non-isothermal stretching surface. Nucl. Eng. Des. 241, 4835-4839 (2011)

9. Wolfgang, C.W., Ostwald, W.: An Introduction to Theoretical and Applied Colloid Chemistry, the World of Neglected Dimensions, 1st edn. Stanpobe Press, Boston (1917)

10. Eastman, J.A., Choi, S.U.S., Li, S., Yu, W., Thompson, L.J.: Anomalously increased effective thermal conductivities of ethylene glycol-based nanofluids containing copper nanoparticles. Applied Phys. Letters. 78, 718-720 (2001)

11. Liu, M.S., Lin, M.C.C., Huang, I.T., Wang, C.C.: Enhancement of thermal conductivity with CuO for nanofluids. Chem. Eng. Technol. 29, 72-77 (2006)

12. Garg, J., Poudel, B., Chiesa, M., Gordon, J.B., Ma, J.J., Wang, J.B., Ren, Z.F., Kang, Y.T., Ohtani, H., Nanda, J., McKinley, G.H., Chen, G.: Enhanced thermal conductivity and viscosity of copper nanoparticles in ethylene glycol nanofluid. J. Appl. Phys. 103, 074301-074306 (2008)

13. S.U.S. Choi, Enhancing Thermal Conductivity of Fluids with Nanoparticles, in: The Proceedings of the 1995 ASME Int. Mech. Eng. Congress and Exposition, San Francisco, ASME, FED 231/MD 66 (1995) 99-105

14. Choi, S.U.S., Zhang, Z.G., Yu, W., Lockwood, F.E., Grulke, E.A.: Anomalously thermal conductivity enhancement in nanotube suspensions. Applied Phys. Letters. 79, 2252-2254 (2001)

15. Yu, W.H., France, D.M., Routbort, J.L., Choi, S.U.S.: Review and comparison of nanofluid thermal conductivity and heat transfer enhancements. Heat Transfer Engineering. 29, 432-460 (2008)

16. Kakaç, S., Pramuanjaroenkij, A.: Review of convective heat transfer enhancement with nanofluids. Int. J. Heat Mass Transf. 52, 3187-3196 (2009)

17. Godson, L., Raja, B., Mohan Lal, D., Wongwises, S.: Enhancement of heat transfer using nanofluids - an overview. Renew. Sustain. Energy Rev. 14(2), 629-641 (2009)

18. Heris, S., Etemad, S.G., Esfahany, M.: Experimental investigation of oxide nanofluids laminar flow convective heat transfer. Int. Commun. Heat Mass Transfer. 33(4), 529-535 (2006)

19. Minsta, H.A., Roy, G., Nguyen, C.T., Doucet, D.: New temperature dependent thermal conductivity data for water based nanofluids. Int. J. Thermal Sci. 48, 363-371 (2009)

20. Daniel, Y.S., Abdul Aziz, Z., Ismail, Z., Salah, F.: Double stratification effects on unsteady electrical MHD mixed convection flow of nanofluid with viscous dissipation and Joule heating. J. Applied Research and Tech. 15(5), 464-476 (2017)

21. Reddy, P.S., Chamkha, A.J., Al-Mudhaf, A.: MHD heat and mass transfer flow of a nanofluid over an inclined vertical porous plate with radiation and heat generation/absorption. Adv. Powder Technol. 28(3), 1008-1017 (2017)

22. Prabhavathi, B., Reddy, P.S., Vijaya, R.B., Chamkha, A.J.: MHD boundary layer heat and mass transfer flow over a vertical cone embedded in porous media filled with Al2O3-water and Cu-water nanofluid. J. Nanofluids. 6, 883-891 (2017)

23. Sreedevi, P., Reddy, P.S., Rao, K.V.S.N., Chamkha, A.J.: Heat and mass transfer flow over a vertical cone through nanofluid saturated porous medium under convective boundary condition with suction/injection. J. Nanofluids. 6, 478-486 (2017)

24. Hayat, T., ljaz Khan, M., Waqas, M., Alsaedi, A., Farooq, M.: Numerical simulation for melting heat transfer and radiation effects in stagnation point flow of carbon-water nanofluid. Comput. Methods Appl. Mech. Eng. 315, 1011-1024 (2017)

25. Madhu, M., Kishan, N., Chamkha, A.J.: Unsteady flow of a Maxwell nanofluid over a stretching surface in the presence of magnetohydrodynamic and thermal radiation effects. Propulsion Power Res. 6(1), 31-40 (2017)

26. Hayat, T., Muhammad, T., Shehzad, S.A., Alsaedi, A.: An analytical solution for magnetohydrodynamic Oldroyd-B nanofluid flow induced by a stretching sheet with heat generation/absorption. Int. J. Thermal Sciences. 111, 274-288 (2017)

27. Hsiao, K.-L.: Micropolar nanofluid flow with MHD and viscous dissipation effects towards a stretching sheet with multimedia feature. Int. J. Heat and Mass Transfer. 112, 983-990 (2017)

28. Eldabe, N.T., Abou-zeid, M.Y.: Homotopy perturbation method for MHD pulsatile non-Newtonian nanofluid flow with heat transfer through a non-Darcy porous medium. J. Egyptian Math. Soc. 25, 375-381 (2017)

29. Sreedevi, P., Reddy, P.S., Chamkha, A.J.: Heat and mass transfer analysis of nanofluid over linear and non- linear stretching surface with thermal radiation and chemical reaction. Powder Technol. 315, 194-204 (2017)

30. Sreedevi, P., Reddy, P.S., Chamkha, A.J.: Magneto-hydrodynamics heat and mass transfer analysis of single and multi-wall carbon nanotubes over vertical cone with convective boundary condition. Int. J. Mech. Sci. 135, 646-655 (2018)

31. Jyothi, K., Reddy, P.S., Reddy, M.S.: Influence of magnetic field and thermal radiation on convective flow of SWCNTswater and MWCNTs-water nanofluid between rotating stretchable disks with convective boundary conditions. Powder Technol. 331, 326-337 (2018) 
32. Zeeshan, A., Shehzad, N., Ellahi, R.: Analysis of activation energy in Couette-Poiseuille flow of nanofluid in the presence of chemical reaction and convective boundary conditions. Results in Phys. 8, 502-512 (2018)

33. Tiwari, R.K., Das, M.K.: Heat transfer augmentation in a two-sided lid-driven differentially heated square cavity utilizing nanofluids. Int. J. Heat Mass Transf. 50, 2002-2018 (2007)

34. Abu-Nada, E.: Application of nanofluids for heat transfer enhancement of separated flows encountered in a backward facing step. Int. J. Heat Fluid Flow. 29, 242-249 (2008)

35. Oztop, H.F., Abu-Nada, E.: Numerical study of natural convection in partially heated rectangular enclosures filled with nanofluids. Int. J. Heat Fluid Flow. 29, 1326-1336 (2008)

36. Hady, F.M., Ibrahim, F.S., Abdel-Gaied, S.M., Eid, M.R.: Effect of heat generation/absorption on natural convective boundary-layer flow from a vertical cone embedded in a porous medium filled with a non-Newtonian nanofluid. Int. Commun. Heat Mass Transfer. 38, 1414-1420 (2011)

37. Ishak, A., Nazar, R., Pop, I.: Boundary layer flow and heat transfer over an unsteady stretching vertical surface. Meccanica. 44, 369-375 (2009)

38. Grubka, L.J., Bobba, K.M.: Heat transfer characteristics of a continuous, stretching surface with variable temperature. ASME J Heat Transfer. 107, 248-250 (1985)

39. Ali, M.E.: Heat transfer characteristics of a continuous stretching surface. Heat Mass Transf. 29, 227-234 (1994)

40. Raju, C.S.K., Sandeep,N., Sulochana, C, Sugunamma, V., Babu, M.J.: Radiation, inclined magnetic field and cross-diffusion effects on flow over a stretching surface. J. Nigerian Math. Soc. 34, 169-180 (2015)

41. Brinkman, H.C.: The viscosity of concentrated suspensions and solution. J. Chem. Phys. 20, 571-581 (1952)

42. Maxwell, J.: A Treatise on Electricity and Magnetism, 2nd edn. Clarendon Press, Oxford (1881)

43. Mahmoud, M.A.A., Megahed, A.M.: Non-uniform heat generation effect on heat transfer of a non-Newtonian power-law fluid over a non-linearly stretching sheet. Meccanica. 47, 1131-1139 (2012)

44. Das, K: Slip flow and convective heat transfer of nanofluids over a permeable stretching surface. Comput. Fluids. 64 34-42 (2012)

\section{Submit your manuscript to a SpringerOpen ${ }^{\circ}$ journal and benefit from:}

- Convenient online submission

- Rigorous peer review

- Open access: articles freely available online

High visibility within the field

- Retaining the copyright to your article 